UCRL-TR-204743

\title{
A Simple Model of Delayed Neutron Emission
}

\author{
by \\ Dermott E. Cullen \\ University of California \\ Lawrence Livermore National Laboratory \\ P.O.Box 808/L-159 \\ Livermore, CA 94550 \\ Tele: 925-423-7359 \\ E.Mail: cullen1@llnl.gov \\ Website: http://www.Ilnl.gov.cullen1
}

June 6, 2004

U.S. Department of Energy

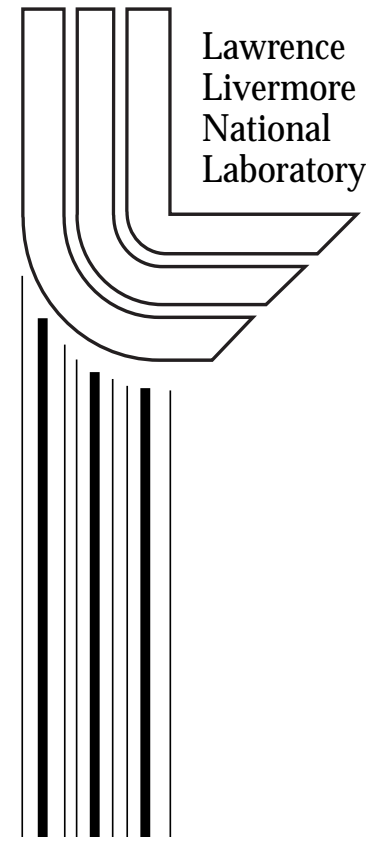

Approved for public release; further dissemination unlimited. 


\section{DISCLAIMER}

This document was prepared as an account of work sponsored by an agency of the United States Government. Neither the United States Government nor the University of California nor any of their employees, makes any warranty, express or implied, or assumes any legal liability or responsibility for the accuracy, completeness, or usefulness of any information, apparatus, product, or process disclosed, or represents that its use would not infringe privately owned rights. Reference herein to any specific commercial product, process, or service by trade name, trademark, manufacturer, or otherwise, does not necessarily constitute or imply its endorsement, recommendation, or favoring by the United States Government or the University of California. The views and opinions of authors expressed herein do not necessarily state or reflect those of the United States Government or the University of California, and shall not be used for advertising or product endorsement purposes.

Work performed under the auspices of the U. S. Department of Energy by the University of California Lawrence Livermore National Laboratory under Contract W-7405-Eng-48.

This report has been reproduced directly from the best available copy.

Available to DOE and DOE contractors from the

Office of Scientific and Technical Information

P.O. Box 62, Oak Ridge, TN 37831

Prices available from (423) 576-8401

http://apollo.osti.gov/bridge/

Available to the public from the National Technical Information Service

U.S. Department of Commerce 5285 Port Royal Rd., Springfield, VA 22161 http://www.ntis.gov/

OR

Lawrence Livermore National Laboratory Technical Information Department's Digital Library http://www.llnl.gov/tid/Library.html 
UCRL-TR-204743

\section{A Simple Model of Delayed Neutron Emission \\ by \\ Dermott E. Cullen \\ University of California \\ Lawrence Livermore National Laboratory \\ P.O.Box 808/L-159 \\ Livermore, CA 94550}

Tele: 925-423-7359

E.Mail: cullen1@llnl.gov

Website: http://www.llnl.gov.cullen1

\section{Overview}

The nuclear data library ENDF/B-VI [1, 2] now includes delayed neutron information for some isotopes. In all cases the ENDF/B-VI delayed neutron model represents the time dependent emission by a sum of six time dependent decay modes, each with its own time constant $[3,4]$,

$P\left(E, E^{\prime}, t\right) d t d E^{\prime}=\left\langle v d(E)>\sum_{k=1}^{6} \mathrm{P}_{\mathrm{k}}{ }^{*} \operatorname{Exp}\left[-\lambda_{\mathrm{k}}{ }^{*} \mathrm{t}\right] \chi_{\mathrm{k}}\left(E^{\prime}\right) d\left[\lambda_{\mathrm{k}}{ }^{*} t\right] d E^{\prime}\right.$

where,

$<v d(E)>$ - delayed yield, which is a function of incident neutron energy.

$\mathrm{P}_{\mathrm{k}} \quad$ - partial yields (the six partial yields sum to unity)

$\lambda_{\mathrm{k}} \quad$ - time constants $\left(\mathrm{sec}^{-1}\right)$

$\chi\left(E^{\prime}\right) \quad$ - energy dependent emission spectra (normalized to unity integrated over $E^{\prime}$ )

Integrated over secondary energy E', we can see the time dependence,

$P(E, t) d t=\left\langle v d(E)>\sum_{k=1}^{6} \mathrm{P}_{\mathrm{k}} * \lambda_{\mathrm{k}}{ }^{*} \operatorname{Exp}\left[-\lambda_{\mathrm{k}}{ }^{\mathrm{t}}\right] \mathrm{dt}\right.$

Integrated over time t, we can see the initial and secondary energy dependence, $P\left(E, E^{\prime}\right) d E^{\prime}=<v d(E)>\sum_{k=1}^{6} \mathrm{P}_{\mathrm{k}} \chi_{\mathrm{k}}\left(E^{\prime}\right) d E^{\prime}$

Integrated over secondary energy E' and over time t, gives the delayed yield,

$P(E)=\langle v d(E)>$ 
The format allows for the partial yields, $\mathrm{P}_{\mathrm{k}}$, and emission spectra, $\chi_{\mathrm{k}}\left(E^{\prime}\right)$, to vary with incident neutron energy, but currently in all cases these are defined as independent of the incident neutron energy. Therefore, currently the only dependence on incident neutron energy is through the delayed neutrons per fission, $<v d(E)>$.

\section{When can these Data be Used?}

The time constants in ENDF/B-VI are all roughly in the range 0.01 and $3 \mathrm{sec}^{-1}$, corresponding to short delay times, and they should only be used for short delay times on the order of up to hundreds of seconds. Also there is no correspondence between the decay modes and any specific fission products, or even lumped fission products. Therefore these data cannot be used to solve problems involving fission product burn-up, which requires both decay data and absorption or burn-up data for the fission product; see details below. These data can only be used in the case described above where subsequent to the creation of the fission products, all of the delayed neutrons will over time eventually be emitted by these fission products; none of the fission products will be burned-up before they emit delayed neutrons.

It is important to note that this is consistent with the commonly used practice when solving time independent transport problems to ignore the time dependence of the delayed neutrons and treat them as ALL emitted promptly. Only in the case where burnup of the fission fragments is insignificant will ALL of the delayed neutrons actually be eventually emitted; otherwise the fragments may burn-up before they have a chance to decay and emit delayed neutrons, reducing the number of delayed neutrons actually emitted, which would invalidate the common assumption.

\section{A Simplified Model}

Here I present a model that uses the energy dependent delayed neutrons per fission, partial yields and time constants, to reproduce the exact time dependence defined by the given ENDF/B data. The only approximation to the ENDF/B data that I introduce is a simplified model for the energy dependent emission spectra. My objective is to have a model that is simple, fast and accurate to sample in a Monte Carlo calculation. The model I introduce here conserves the average energy of the neutrons emitted in each of the six decay modes, but does not include all of the detail of the ENDF/B-VI defined emission spectra. This model is also designed to void some problems that can occur when the ENDF/B-VI detailed emission spectra are used in applications, as explained below.

\section{ENDF/B-VI}

ENDF/B-VI includes fission data for 43 isotopes and one isomer. Of these 43 isotopes it includes delayed neutron information for 29 isotopes; there is no delayed neutron data for the remaining 14 isotopes and one isomer. Only neutron emission, not photon emission or anything else, is described by the ENDF/B-VI delayed neutron data. ENDF/B-VI includes complete delayed neutron information for 21 isotopes, namely listing the ZA of each isotope, 
90232, 91231, 92233, 92234, 92235, 92236, 92237, 92238, 93237, 93238, 94238, 94239, 94240, 94241, 94242, 95241, 95242, 95243, 96242, 98249, 98251

This included energy dependent, $\langle v d(E)\rangle$, time constants, $\lambda_{\mathrm{k}}$, partial yields, $\mathrm{P}_{\mathrm{k}}$, and energy dependent emission spectra, $\chi\left(E^{\prime}\right)$; this includes all of the data required for the model of delayed neutron emission described above. Those in red are also included in the ENDL neutron data library described below.

In addition it includes partial delayed neutron information for 8 isotopes, namely listing the ZA of each isotope,

91232, 92232, 93236, 94236, 96243, 96245, 96246, 97249

This included energy dependent, $\left\langle v d(E)>\right.$, time constants, $\lambda_{\mathrm{k}}$, BUT NO partial yields, $\mathrm{P}_{\mathrm{k}}$, or energy dependent emission spectra, $\chi\left(E^{\prime}\right)$; the time dependence of delayed neutrons cannot be calculated from only this information. Those in blue are also included in the ENDL neutron data library described below.

\section{ENDL}

ENDL [5] includes 38 fissile isotopes, namely listing the ZA of each isotope,

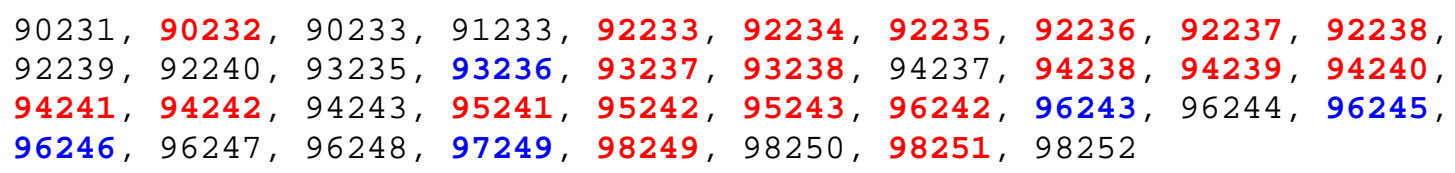

Those in red include complete delayed neutron data in ENDF/B-VI, and those in blue include partial delayed neutron data in ENDF/B-VI. ENDL includes the energy dependent, $\langle v d(E)>$, for all 38 isotopes, but no information defining the time or energy dependence of delayed neutron emission.

\section{Equivalent Data}

My objective is to develop a simple model that can be used with all of the isotopes included in ENDL with the Monte Carlo transport code TART [6, 7]. ENDL currently includes energy dependent, $\langle v d(E)>$ for all isotopes, but have no information defining the time and energy dependence of the emitted neutrons. For those ENDF/B-VI evaluations that include complete data I will use it with ENDL; fortunately data are included in ENDF/B-VI for all of the major isotopes. For each of the remaining isotopes in ENDL I will use ENDF/B-VI data from a similar isotope; similar in the sense of nearest odd-odd, odd-even, even-odd or even-even isotope. For $Z=96,97$, and 98, there are not enough data available to use parity to select data; in these cases all of the isotopes for each $\mathrm{Z}$ were assigned the same data. Below is a table of all the equivalences that I used. 


\begin{tabular}{|ll|ll|ll|}
\hline ENDL & ENDF/B-VI & ENDL & ENDF/B-VI & ENDL & ENDF/B-VI \\
90231 & $\mathbf{9 2 2 3 3}$ & $\mathbf{9 3 2 3 6}$ & $\mathbf{9 3 2 3 8}$ & $\mathbf{9 6 2 4 6}$ & $\mathbf{9 6 2 4 2}$ \\
90233 & $\mathbf{9 2 2 3 3}$ & 94237 & $\mathbf{9 4 2 3 9}$ & 96247 & $\mathbf{9 6 2 4 2}$ \\
91233 & 93237 & 94243 & $\mathbf{9 4 2 4 1}$ & 96248 & $\mathbf{9 6 2 4 2}$ \\
92239 & $\mathbf{9 2 2 3 7}$ & $\mathbf{9 6 2 4 3}$ & $\mathbf{9 6 2 4 2}$ & $\mathbf{9 7 2 4 9}$ & $\mathbf{9 6 2 4 2}$ \\
92240 & $\mathbf{9 2 2 3 8}$ & 96244 & $\mathbf{9 6 2 4 2}$ & 98250 & $\mathbf{9 8 2 5 1}$ \\
93235 & $\mathbf{9 3 2 3 7}$ & $\mathbf{9 6 2 4 5}$ & $\mathbf{9 6 2 4 2}$ & 98252 & $\mathbf{9 8 2 5 1}$ \\
\hline
\end{tabular}

Table. 1: Isotope Equivalence Table

To complete the TART treatment of fission I have also defined delayed neutron parameters for the important spontaneously fissioning isotopes: Pu240, Cm242, Cm242 and Cf252, which can be used in TART as neutron sources for neutron correlation studies.

In summary, by using the existing ENDF/B delayed neutron data for the same isotopes in ENDL, and using the above equivalences I have defined delayed neutron data for all 38 fissile isotopes in ENDL, and these data have now been implemented for use in the TART Monte Carlo transport code.

\section{Simplified Emission Spectra}

For each isotope in ENDF/B-VI spectra are given for each of the six time dependent decay modes. The spectra vary in detail from one isotope to another, but in general are quite similar. Below I show the six spectra for $\mathrm{U}^{235}$, on the left using log-log scale, and on the right using linear-log scale. In all cases the six ENDF/B-VI spectra are defined as a histogram, where each spectrum is constant between successive tabulated values. Each spectrum has a fair amount of detail, and all eventually exponentially decrease at higher energy. Compared to the prompt fission spectra, the delayed spectra are much softer, with average energies roughly in the 400 to $600 \mathrm{keV}$ range. In the simple model that I will use it is this difference in average energy that I will conserve, since it is this which can most easily be seen to effect the results of our transport calculations. 

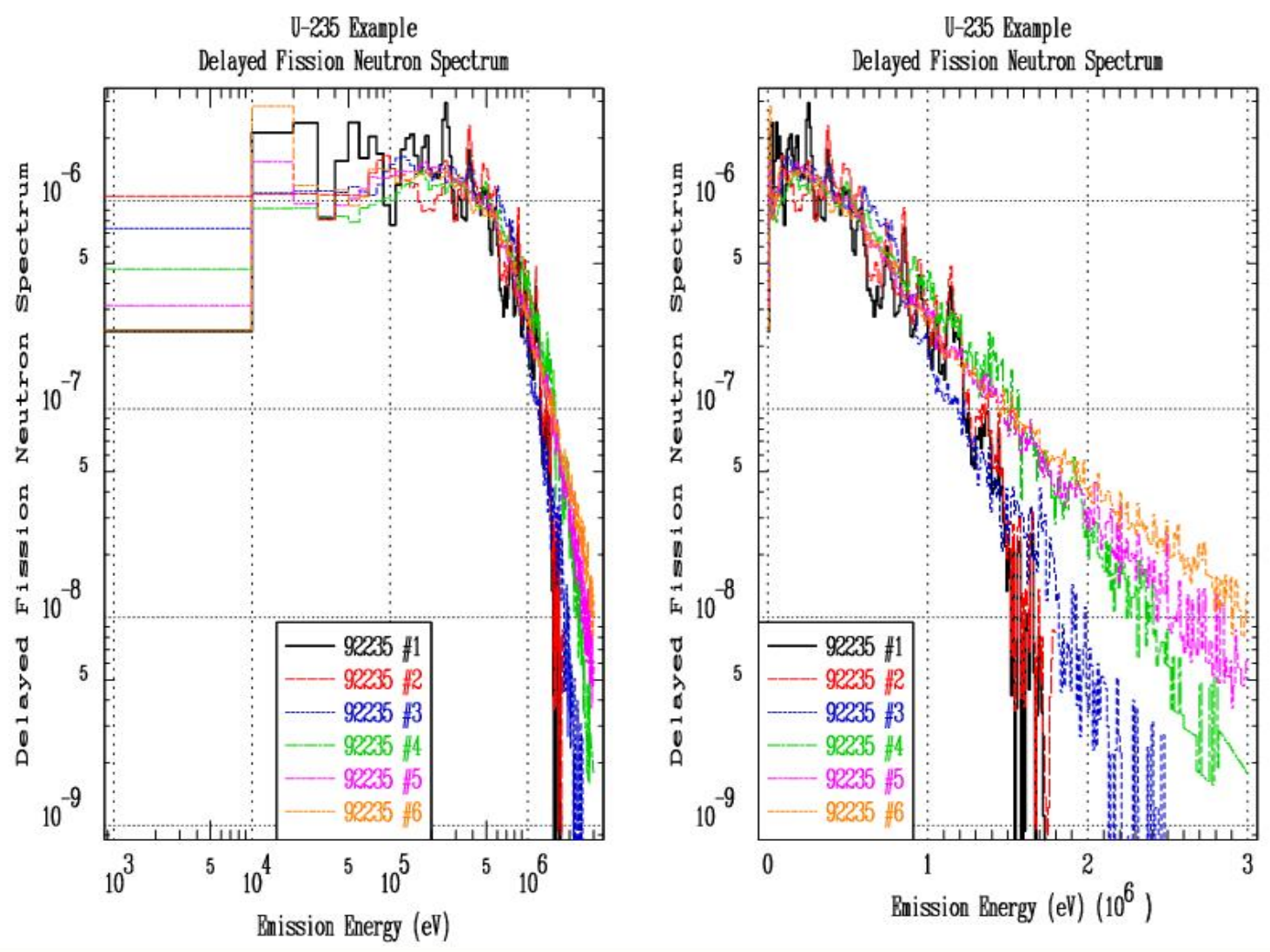

Again, I will mention that the current ENDF/B delayed spectra are in all cases independent of the incident neutron energy. For example, the data shown above are the only spectra provided for $\mathrm{U}^{235}$, independent of the incident neutron energy.

I will assume that each spectrum corresponds to the emission of a neutron from a single, stationary fission product. In this case the lowest order assumption is that the neutrons are emitted in a Maxwellian distribution,

$\mathrm{S}(\mathrm{E}) \mathrm{dE}=\mathrm{C} *[\mathrm{E} / \mathrm{T}]^{1 / 2} * \operatorname{Exp}[-\mathrm{E} / \mathrm{T}] \mathrm{dE}, \quad$ Average Energy $<\mathrm{E}>=(3 / 2) \mathrm{T}$

I have fit each ENDF/B-VI distribution to a Maxwellian to conserve the average energy of the emitted neutrons. Below are the results for each of the six U235 spectra; results for all other isotopes are very similar and will not be shown here. 

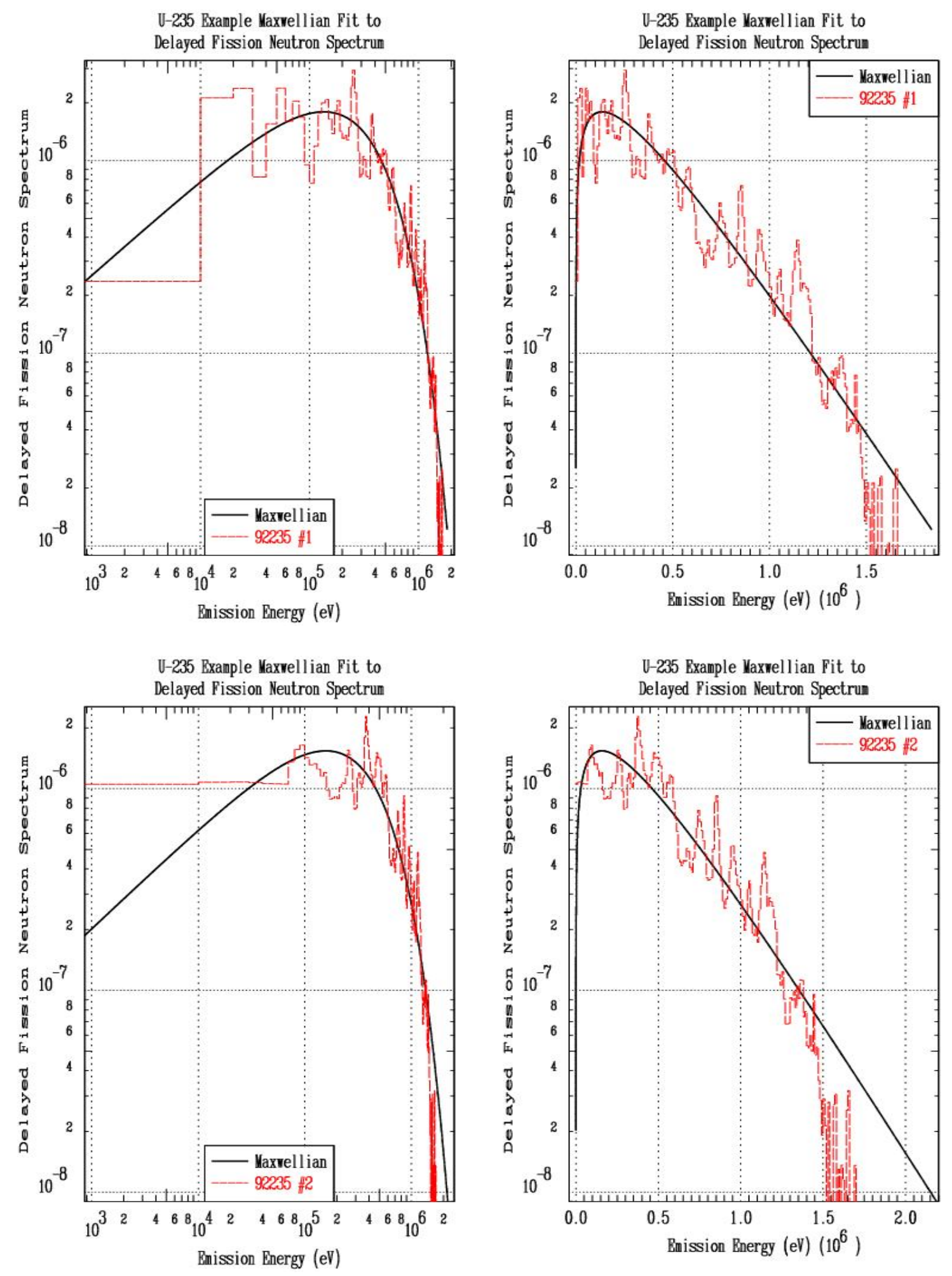

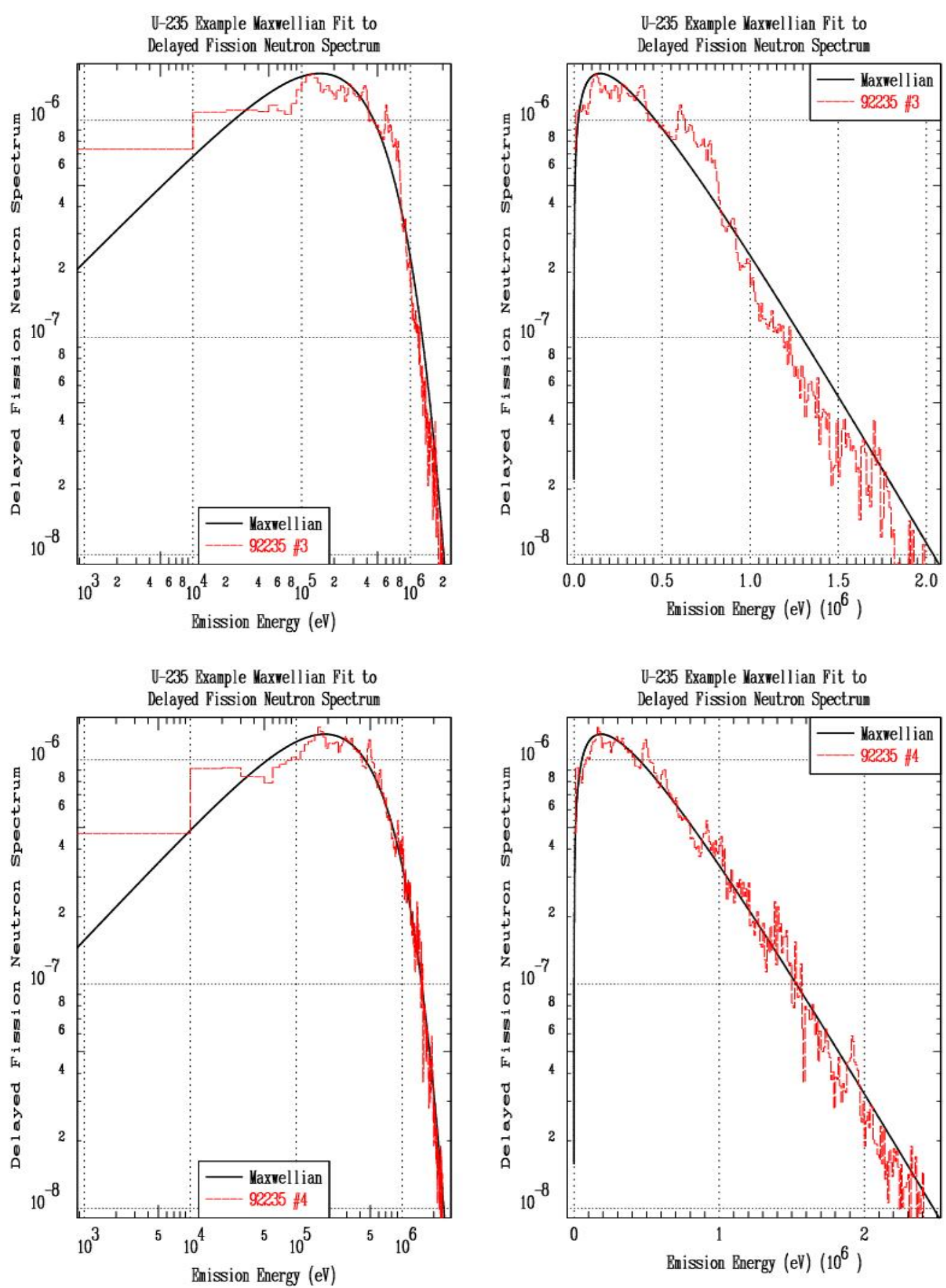

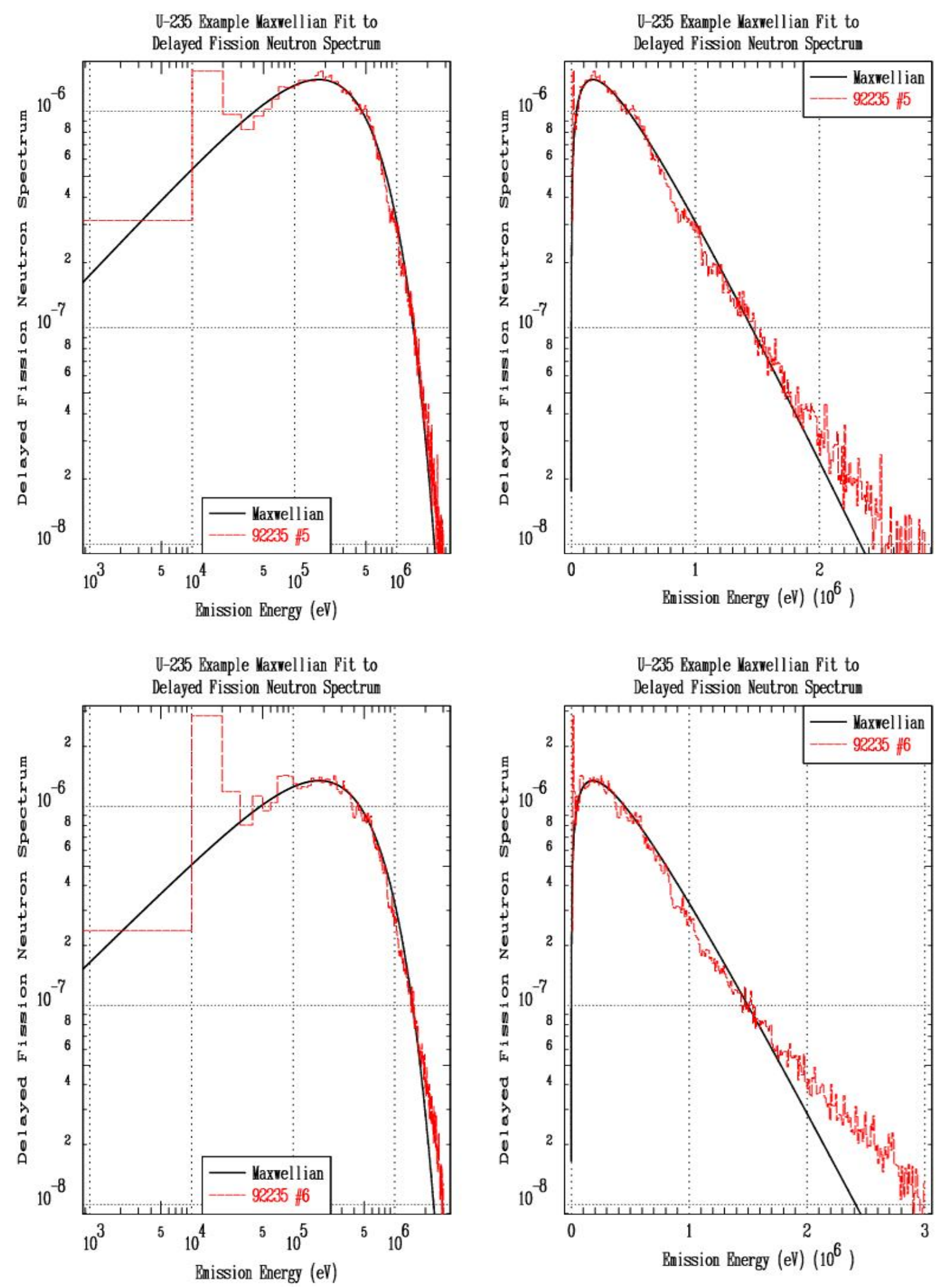

These fits may seem to be quite crude, but it is important to realize that the emitted delayed neutrons are usually only a fraction of one per-cent of the total number of 
neutrons emitted per fission, and that the uncertainties in these delayed neutron spectra are quite large; an exception is $\mathrm{U}^{238}$, which has an unusually large delayed fraction. Also be aware that the histogram representation of the delayed spectra as given in ENDF/B-VI can cause problems when used in applications. For example, each of the ENDF/B-VI delayed spectra is defined by a constant value between 0 and $10 \mathrm{keV}$, which results in the completely unrealistic low energy variation of the ENDF/B-VI spectra shown in the above figures. In this case, rather than being a crude approximation to reality, I judge the low energy square root of $E$ ' variation of the Maxwellian to be a much better approximation to reality than the constant value given in ENDF/B-VI; see below for more details.

In ENDF/B-VI for $\mathrm{U}^{235}$ at thermal energy $(0.0253 \mathrm{eV})$, the total neutrons emitted per fission is defined as 2.4367 and the delayed 0.0167, so that the prompt is the difference, 2.4200. The delayed fraction is 0.00685 (0.0167/2.4367), i.e., of all neutrons emitted in fission 0.00685 are delayed. For example, in a Monte Carlo calculation if you sample 1 million neutrons produced by fission only 6850 will be delayed, and these will be distributed in secondary energy, time and direction. This may seem like a very small amount that would not even be seen in a combined spectrum of prompt plus delayed emission, but actually because the delayed spectra have a much lower average energy they are concentrated over a smaller energy interval and are larger than you might think. To illustrate this point below I show a comparison of prompt and delayed $\mathrm{U}^{235}$ spectra, in this case both are normalized to unity (not the number of neutrons actually emitted in each). In this case the average energy is $2.03 \mathrm{MeV}$ for prompt and $405 \mathrm{keV}$ for delayed. Because the delayed emission is concentrated over a smaller energy interval, at its peak and at lower energies it is roughly ten times higher than the prompt emission. Remember this is for spectra both normalized to unity, but it illustrates why the effect of delayed neutrons on the total fission neutron spectra is more noticeable than you would suspect based on the 0.00685 delayed fraction.
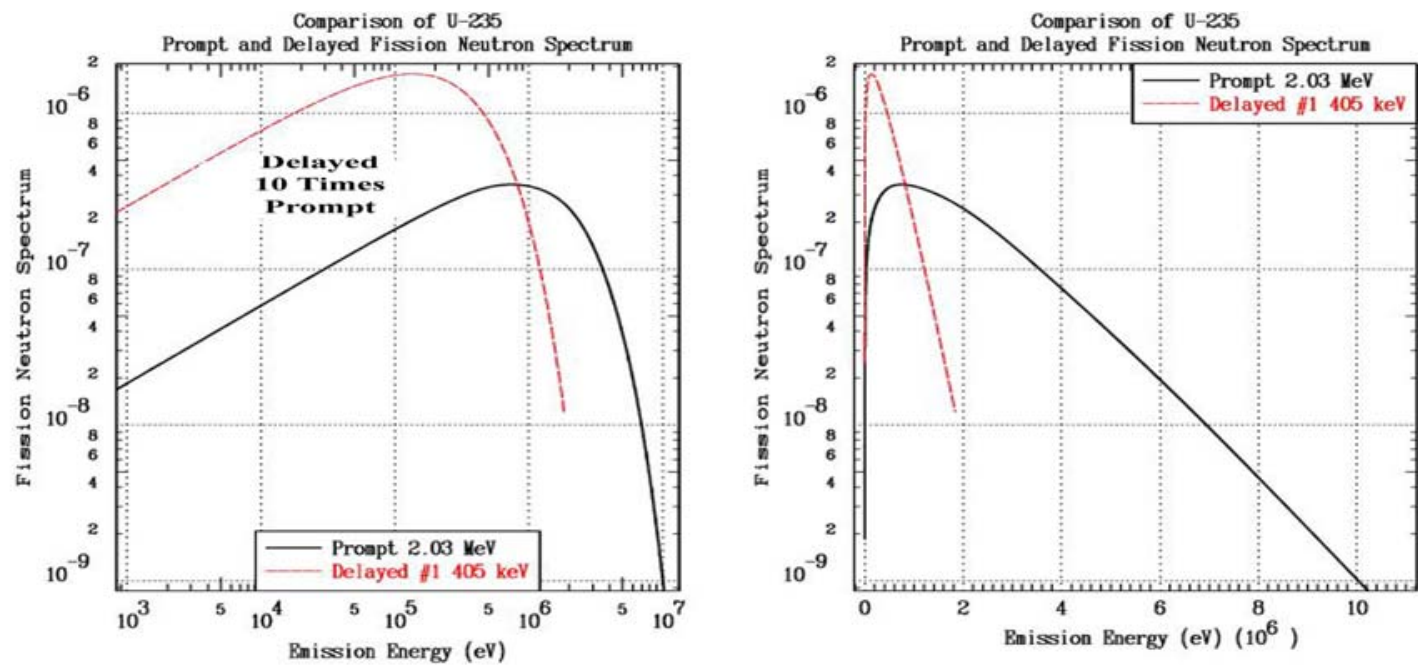
Below I show a comparison for $\mathrm{U}^{235}$ of the sum of the six spectra using my Maxwellian approximation and the original ENDF/B-VI data. Both spectra are normalized to the delayed yield of 0.0167 neutrons per fission. In ENDF/B-VI the prompt neutron emission spectra are given at secondary energies, E', $10 \mathrm{eV}$ and above, so that the below figures also show the energy range of $10 \mathrm{eV}$ and above.

From the figure on the left, we can clearly see the problem of using the original histogram defining the yield as constant between 0 and $10 \mathrm{keV}$; the constant yield below $10 \mathrm{keV}$ is completely unrealistic. From the figure on the right we can see that at higher energies the sum of the six Maxwellians is an excellent approximation to the sum of the six original histogram spectra over three orders of magnitude in $\chi\left(E^{\prime}\right)$.
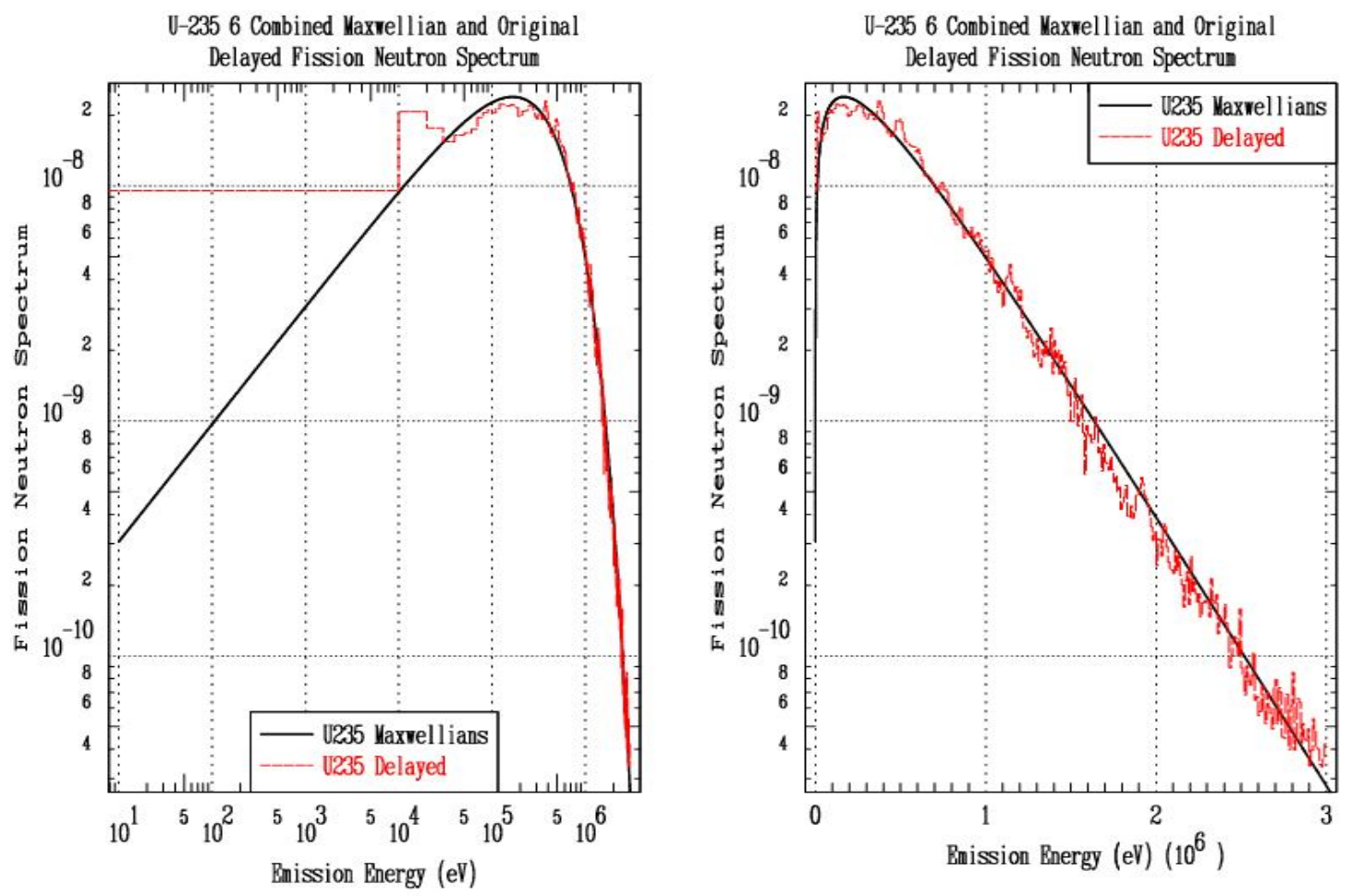


\section{Results Using the ENDF/B-VI Data}

Below I show the prompt and total neutron emission spectra using the ENDF/B-VI prompt continuous spectrum and the delayed histogram data. Note the effect of the unrealistic delayed low energy constant (histogram) data between 0 and $10 \mathrm{keV}$; the total yield begins to deviate from the prompt below roughly $1 \mathrm{MeV}$, on the below figure we can start to see this by $10 \mathrm{keV}$, and at lower energies it grows to over three times (over $300 \%$ ) the prompt emission by $10 \mathrm{eV}$.
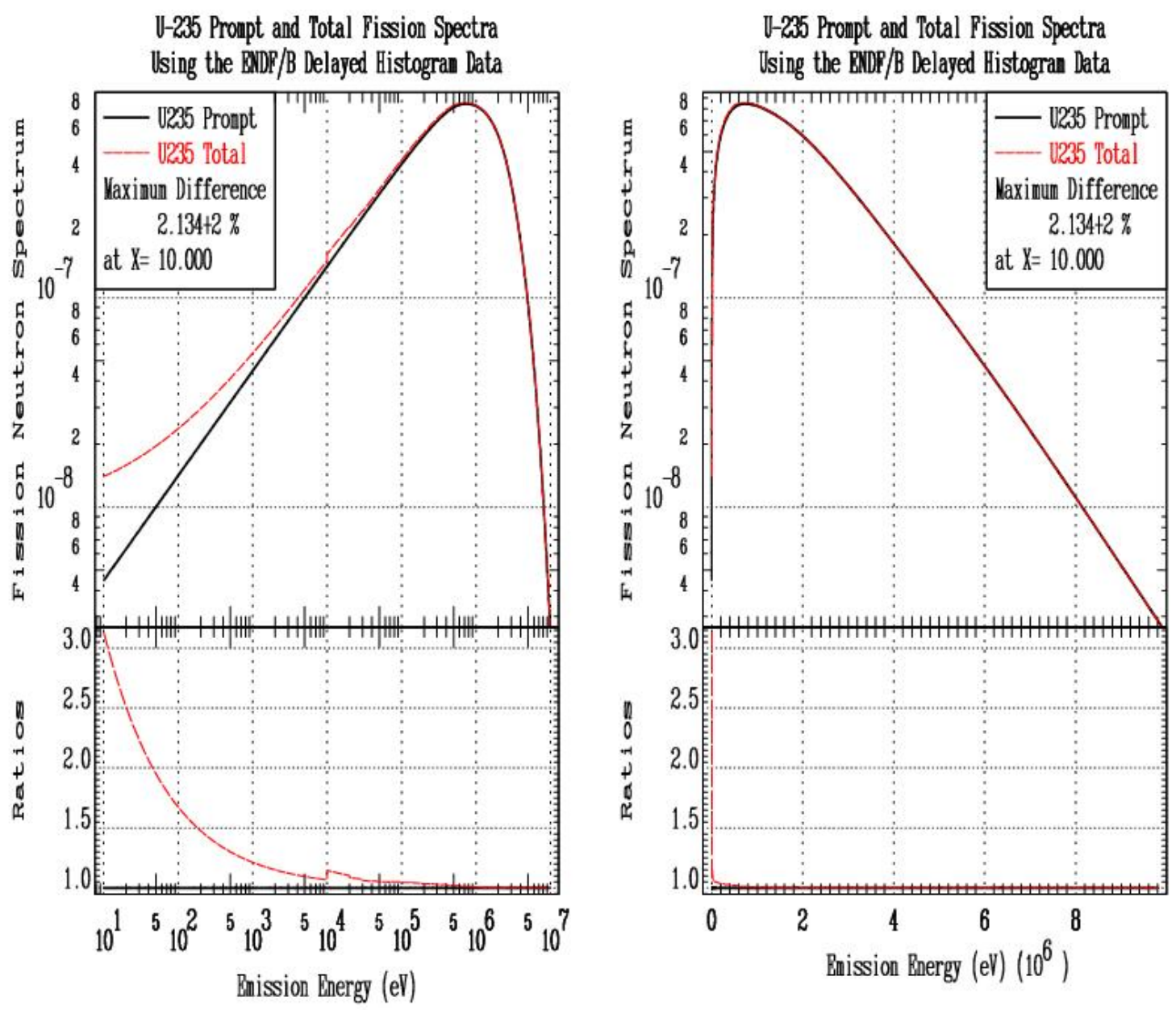


\section{Results Using Maxwellian Delayed Spectra Model}

Below I show the prompt and total neutron emission spectra using the ENDF/B-VI prompt continuous spectrum and my proposed Maxwellian delayed spectra. Note that both prompt and delayed spectra now have a square root of E' variation at low energy. The total spectrum smoothly varies from the prompt spectra below about $1 \mathrm{MeV}$ and reaches a constant ratio roughly $6.85 \%$ higher than the prompt at lower energy. Recalling that the delayed fraction is 0.00685 , but that the concentration of the delayed spectra over a smaller energy range increases its magnitude at roughly a factor of ten, the $6.85 \%$ rise of the total spectra relative to the prompt is exactly what we physically expect.
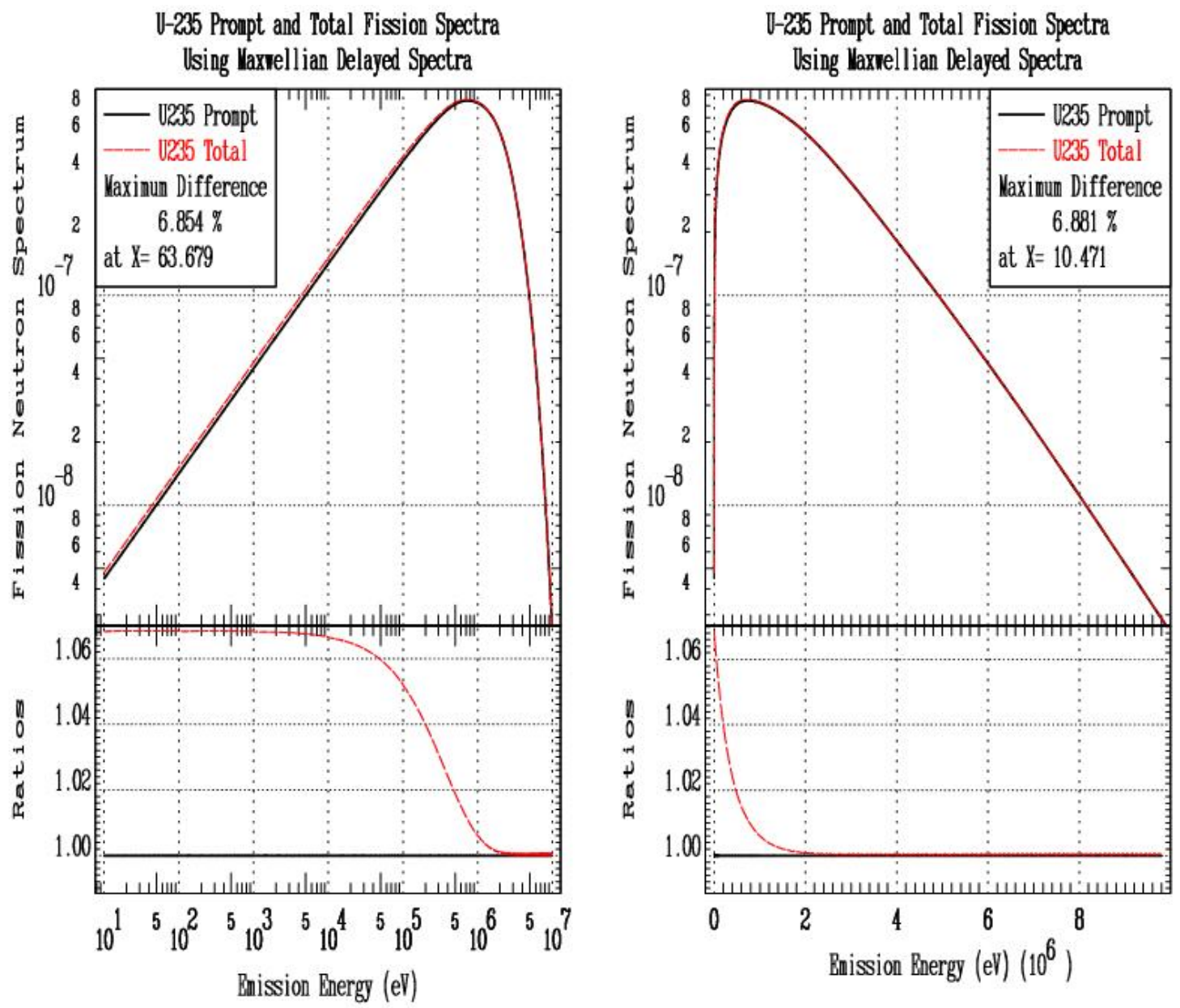


\section{Point-Kinetics and In-Hour Equations}

The point-kinetics and in-hour equations can be derived from the linear Boltzmann equation that we solve using neutron transport codes. This involves integrating over all space (point-kinetics) or all space and energy (in-hour). It is informative to derive these equations because, hopefully, it clearly shows the approximations involved in using the ENDF/B-VI data, and the limited range of problems that we can practically consider accurately solving. Starting from the Boltzmann equation,

$$
\begin{array}{ll}
\frac{1}{v} \frac{\partial N}{\partial t}+\Omega * \nabla N+\Sigma t^{*} N=\iiint F\left(r, \Omega^{\prime}, E^{\prime}, t^{\prime}->\Omega, E, t\right) N\left(r, \Omega^{\prime}, E^{\prime}, t^{\prime}\right) d \Omega^{\prime} d E^{\prime} d t^{\prime}+S \\
N(r, \Omega, E, t) & \text { - neutron flux } \\
v & \text { - neutron speed (speed, not velocity) } \\
\Sigma t(r, E, t) & \text { - total cross section } \\
F & \text { - transfer from } \Omega^{\prime}, E^{\prime} \cdot t^{\prime} \text { to } \Omega, E, t \\
S(r, \Omega, E, t) & \text { - extraneous (flux independent) source }
\end{array}
$$

We subdivide the transfer by reaction, into scatter (inelastic and inelastic), and neutron production; for simplicity here I only explicitly include production due to fission, but in practice this should also include production due to (n,2n), (n,3n), etc.

$$
\begin{aligned}
F= & \sum \operatorname{scatter}\left(r, E^{\prime}\right) f s\left(\Omega^{\prime}, E^{\prime}->\Omega, E\right)+ \\
& <v p\left(E^{\prime}\right)>\Sigma \text { fission }\left(r, E^{\prime}\right) f p\left(\Omega^{\prime}, E^{\prime}->\Omega, E\right)+ \\
& <v d\left(E^{\prime}\right)>\Sigma f i s \operatorname{sion}\left(r, E^{\prime}\right) f d\left(\Omega^{\prime}, E^{\prime}, t^{\prime}->\Omega, E, t\right)
\end{aligned}
$$

Here we assume that all neutrons outgoing from reactions appear instantaneously at the time each reaction occurs (no time dependence), except for delayed neutrons, where a fission at an earlier time t' can result in the emission of a neutrons at a later time $t$. We can simplify this time dependence by realizing that the delayed neutrons are emitted by specific fission products; the neutron emission is delayed until the specific fission product decays, usually by beta emission. This can potentially simplify the problem because rather than having to know the flux at all earlier times as a function of space, energy, direction, and time, we need only know the precursor concentrations as a function of space and time. The equation defining the concentration of any specific fission product is defined by,

$$
\frac{\partial C k}{\partial t}=-\lambda_{\mathrm{k}} C k(r, t)-C k(r, t) \int \sigma_{\mathrm{k}}\left(E^{\prime}\right) * N\left(r, E^{\prime}, t\right) d E^{\prime}+\int<v d k>\Sigma f * N\left(r, E^{\prime}, t\right) d E^{\prime}
$$

We also need an initial condition defining $C k(r, 0)$. The three terms on the right hand side of this equation correspond to,

$$
\int<v d k>\Sigma f * N\left(r, E^{\prime}, t\right) d E^{\prime} \quad \text { - creating of fission product due to fission }
$$




$$
\begin{aligned}
& C k(r, t) \int \sigma_{\mathrm{k}}\left(E^{\prime}\right) * N\left(r, E^{\prime}, t\right) d E^{\prime} \text { - burn-up of the fission product } \\
& \lambda_{\mathrm{k}} C k(r, t) \\
& \text { - decay of the fission product }
\end{aligned}
$$

In the case of the ENDF/B-VI data we do not know the burn-up cross section, $\sigma_{\mathrm{k}}\left(E^{\prime}\right)$, for the fission products; again, the ENDF/B-VI delayed neutron data are not associated with any specific fission product or even lumped fission productions. Therefore we cannot use the ENDF/B-VI data in applications where there is significant fission product burn-up during the application; we simply do not have the data required to model this. Therefore we are limited to modeling the concentration of fission product by,

$$
\frac{\partial C k}{\partial t}=-\lambda_{\mathrm{k}} C k(r, t)+\int<v d k>\Sigma f * N\left(r, E^{\prime}, t\right) d E^{\prime}
$$

Solving for $C k(r, t)$,

$$
\begin{aligned}
C k(r, t)= & C k(r, t 0) \operatorname{Exp}\left[-\lambda_{\mathrm{k}}(t-t 0)\right]+ \\
& \int_{t 0}^{t} \operatorname{Exp}\left[-\lambda_{\mathrm{k}}\left(t^{\prime}-t 0\right)\right] d t^{\prime} \int<v d k>\Sigma f * N\left(r, E^{\prime}, t^{\prime}\right) d E^{\prime}
\end{aligned}
$$

Here we can see that the fission product precursor concentration $C k(r, t)$ depends on the initial condition $C k(r, t 0)$ and the neutron flux between the initial and current times. Note that as time progresses $C k(r, t)$ becomes independent of the initial condition, i.e., its contributions is exponentially decreasing.

If we had a time independent flux this predicts that the fission product concentrate would approach a steady state, where the decay of the fission product would balance the creation of fission products due to fission,

$$
C k(r)=\int<v d k>\Sigma f * N\left(r, E^{\prime}\right) d E^{\prime} / \lambda_{\mathrm{k}}
$$

In the other extreme if we had a steady state distribution and could suddenly somehow turn off the flux at time t0, the fission product concentration would exponentially decrease at subsequent times,

$$
C k(r, t)=C k(r, t 0) * \operatorname{Exp}\left[-\lambda_{\mathrm{k}}(t-t 0)\right]
$$

In practice this is not easy to do since the decay of the fission products creates delayed neutrons, which means flux (so you cannot simply turn it off). However, with a time dependent source we can approximate this condition.

It is the decay of the fission products that results in the delayed neutrons, 
$<v d(E)>\Sigma$ delayed $\left(r, \Omega^{\prime}, E^{\prime}, t^{\prime}->\Omega, E, t\right) N=<v d(E)>\sum_{k} \chi_{\mathrm{k}}\left(E^{\prime}\right) * \lambda_{\mathrm{k}} * C_{\mathrm{k}}(r, t)$

Since we cannot consider fission product burn-up this is equivalent to the fission product model described above; here I reverse the prime and unprimed variables, so that transfer is from the initial E', t' to the final E, t (sorry about that),

$$
P\left(E^{\prime}, E, t^{\prime}, t\right) d t d E=\left\langle v d\left(E^{\prime}\right)>\sum_{k=1}^{6} \mathrm{P}_{\mathrm{k}} * \lambda_{\mathrm{k}}{ }^{*} \operatorname{Exp}\left[-\lambda_{\mathrm{k}}{ }^{*}\left(\mathrm{t}-\mathrm{t}^{\prime}\right)\right] \chi_{\mathrm{k}}(E) d t d E\right.
$$

\section{Delayed Neutrons Change the Problem and How we Solve It}

When we solve the Boltzmann equation without delayed neutrons everything is assumed to happen instantaneously. We can start a calculation at any arbitrary initial time and without knowing anything about the system at earlier times we can solve the problem for any subsequent time.

With delayed neutrons this is no longer true, since at least in principle the flux at any time is now coupled to the flux at ALL earlier times. In some cases the use of the equations defining the fission product concentrations allows us to simplify this coupling by requiring only that we know the initial concentration of fission products $C k(r, 0)$. This is the procedure normally followed in solving point-kinetics or the in-hour equation, where space is not a variable.

Unfortunately this is not practical for use in our Monte Carlo calculations because in general we do not know the spatial distribution of the fission products. The spatial distribution of fission products at any given initial time depends on the spatially dependent neutron flux at all earlier times, which is unknown at the beginning of our calculation and is what we want to calculate.

For convenience I will use neutron density, $\mathrm{n}$, rather than neutron flux, $\mathrm{N}=\mathrm{n} * \mathrm{v}$ in the following. If we integrate the Boltzmann equation over all space, directions, and energy, remembering that all quantities are averaged over all variables except time, we find,

$$
\frac{\partial n(t)}{\partial t}+L * v^{*} n(t)+\Sigma t * v * n(t)=F(t)+v^{*} s(t)
$$

Here $\mathrm{L}$ is the leakage from the system.

$$
\begin{aligned}
& F=\Sigma s * v^{*} n(t)+<v p>\Sigma f * v^{*} n(t)+ \\
& \int<v d>\Sigma f * v^{*} n\left(t^{\prime}\right) \sum_{k=1}^{6} \mathrm{P}_{\mathrm{k}}^{*} \lambda_{\mathrm{k}}{ }^{*} \operatorname{Exp}\left[-\lambda_{\mathrm{k}}{ }^{*}\left(\mathrm{t}-\mathrm{t}^{\prime}\right)\right] \mathrm{dt} \mathrm{t}^{\prime}
\end{aligned}
$$

Without delayed neutrons the equation is simply, 


$$
\begin{aligned}
& \frac{\partial n(t)}{\partial t}-\alpha^{*} n(t)=v^{*} s(t) \\
& \begin{aligned}
\alpha & =v^{*}[<v p>\Sigma f-(L+\Sigma t-\Sigma s)]=\text { production - removal } \\
\quad & * v^{*}[<v p>\Sigma f-(L+\Sigma a)]=\text { production - (leakage \& absorption) }
\end{aligned}
\end{aligned}
$$

In this case we have a simple exponential solution.

When delayed neutrons are included there is no simple exponential solution. In this case the equation is,

$$
\frac{\partial n(t)}{\partial t}+\alpha^{*} n(t)=v^{*} s(t)+\int<v d>\Sigma f * v^{*} n\left(t^{\prime}\right) \sum_{k=1}^{6} \mathrm{P}_{\mathrm{k}}^{*} \lambda_{\mathrm{k}} * \operatorname{Exp}\left[-\lambda_{\mathrm{k}}^{*}\left(\mathrm{t}-\mathrm{t}^{\prime}\right)\right] \mathrm{dt} \mathrm{t}^{\prime}
$$

This form indicates that the solution will be a sum of exponentials. In addition in order to solve this equation at time $t$ we must define the flux at ALL earlier times t'.

For use in our Monte Carlo calculations it is not practical for us by define the flux at all previous times as a function of spatial position, and energy. The only practical use of these equations is when we start at a given time, define the flux at all earlier times to be zero, allow the system to evolve over some period of time and then introduce a change in the system to perturb the steady state and observe what subsequently happens to the system. Only in this manner can we accurately simulate the behavior of a system.

The easiest such change is to use a time dependent source, either an instantaneous source or a source over some fixed period of time, then turn off the source and watch the exponential decay of the flux in the system. If there is no fissile material in the system the flux will rapidly exponentially decay, typically in microseconds to milliseconds depending on the system. However, if fissile material is present the decay will be much slower, as delayed neutrons are emitted from fission products over times of milliseconds to seconds. The strength of this long term signal will naturally depend on both the magnitude and duration of neutron source and the concentration of fissile material in the system.

\section{Importance of Energy Dependence}

Since they are called delayed neutrons usually when discussing them the concentration is normally on time. However what should not be overlooked is the important effect of the slower average energy of delayed neutrons compared to prompt neutrons, i.e., roughly $500 \mathrm{keV}$ versus $2 \mathrm{MeV}$. The effect that this has is system dependent. For example, for fast neutron systems, compared to emitting all neutrons in the prompt emission spectrum, emitting delayed neutrons in the softer delayed emission spectrum means that the delayed neutrons are emitted below the effective $\mathrm{U}^{238}$ fission threshold, which can reduce reactivity. In contrast for thermal systems the lower average delayed neutron energy can reduce leakage and absorption during slowing down resulting in a higher reactivity. 


\section{TART Verification: What TART can and cannot Model}

TART is a time dependent Monte Carlo code in the sense that it allows time dependent particles, so that we can model the time dependent emission of delayed neutrons. But TART has fixed geometry that is NOT time dependent, neither are any properties such as composition, density, temperature, etc. Therefore TART cannot model control rod motion, changes in density or temperature of any material, such as water versus steam or anything else that changes the geometry of a problem. This means that there is no feedback such as expansion or contraction, temperature change, fuel or fission product burn-up. In summary: with TART particles being transported can be time dependent, but the medium through which they transport is time independent. This naturally restricts the types of problems involving delayed neutrons that TART can handle, but there are still enough interesting problems that TART can handle to makes it worthwhile correctly modeling delayed neutrons in TART.

This simple model of delayed neutrons has now been implemented in the TART Monte Carlo code. Below I present a range of results first to verify that TART is correctly sampling delayed neutron emission and then to illustrate some problems that can be modeled by TART in which correctly modeling delayed neutrons is important.

\section{Broomstick}

To verify any kinematics model one of the simplest approaches is to perform a broomstick calculation, which is designed to allow each particle to undergo one and only one collision, so that the resulting distribution in secondary energy, direction and time should correspond exactly of the kinematics model being tested.

Here I perform a broomstick calculation using a cylinder of very small radius $\left(10^{-5} \mathrm{~cm}\right)$, and very long length $\left(10^{+5} \mathrm{~cm}\right)$. I use an instantaneous $(\mathrm{t}=0$, only) neutron point source located on the central axis of the cylinder near one end of the cylinder, and monodirectional, directly up the axis of the cylinder. The cylinder contains a fissile material; in this case $\mathrm{U}^{235}$ at 18.7 grams/cc density. With this geometry each neutron is forced to quickly have one and only one event, and following each event all outgoing neutrons from the event change direction and leak from the narrow cylinder into the surrounding volume, where I tally their energy, time and direction. For a thermal neutron incident the only possible events are elastic scatter and fission, but only fission will result in fast neutrons, and only delayed neutrons appear at later time. Therefore by examining the energy and time dependence of the leakage I can determine whether or not the single event delayed neutron kinematics as implemented in TART are performing as intended.

Normalized per incident neutron all leakage will be essentially instantaneous, except for the delayed neutrons, whose time dependent variation is defined in absolute form as,

$<v d>\Sigma f / \Sigma t \sum_{k=1}^{6} \mathrm{P}_{\mathrm{k}} * \lambda_{\mathrm{k}} * \operatorname{Exp}\left[-\lambda_{\mathrm{k}}{ }_{\mathrm{t}}\right]$ 
$\Sigma f / \Sigma t$ defines the probably of fission per collision, $\langle v d\rangle$ defines the number of delayed neutrons per fission, and the remainder defines the time dependence of the six modes. For a source that is constant from $\mathrm{t}=\mathrm{t}$ to $\mathrm{t} 1$, and zero for longer times the solution is merely the above, integrated from $\mathrm{t}=0$ to $\mathrm{t}$, followed by exponential decay,

$<v d>\Sigma f / \Sigma t \sum_{k=1}^{6} \mathrm{P}_{\mathrm{k}}{ }^{*}\left[1-\operatorname{Exp}\left(-\lambda_{\mathrm{k}}{ }^{*} \mathrm{t} 1\right)\right] \operatorname{Exp}\left[-\lambda_{\mathrm{k}} *(\mathrm{t}-\mathrm{t} 1)\right], \mathrm{t}>\mathrm{t} 1$

Finally, the solution integrated over all time is,

$<v d>\Sigma f / \Sigma t \sum_{k=1}^{6} \mathrm{P}_{\mathrm{k}}=<v d>\Sigma f / \Sigma t$

This merely states that all of the delayed neutrons will eventually be emitted.

At room temperature for thermal neutrons the pertinent $\mathrm{U}^{235}$ data includes,

$$
\begin{aligned}
<v d> & =0.0167 \\
\Sigma f & =585.07 \text { barns } \\
\Sigma t & =698.89 \text { barns }
\end{aligned}
$$

\begin{tabular}{|c|c|c|c|}
\hline $\mathrm{k}$ & $\mathrm{Pk}$ & $\lambda \mathrm{k}\left(\mathrm{sec}^{-1}\right)$ & $\mathrm{Tk}(\mathrm{MeV})$ \\
\hline 1 & 0.03500 & $1.3336 \mathrm{~d}-2$ & 0.27021 \\
\hline 2 & 0.18070 & $3.2739 \mathrm{~d}-2$ & 0.31436 \\
\hline 3 & 0.17252 & $1.2078 \mathrm{~d}-1$ & 0.29509 \\
\hline 4 & 0.38678 & $3.0278 \mathrm{~d}-1$ & 0.37162 \\
\hline 5 & 0.15858 & $8.4949 \mathrm{~d}-1$ & 0.34547 \\
\hline 6 & 0.06642 & $2.8530 \mathrm{~d}+0$ & 0.36016 \\
\hline
\end{tabular}

Note, that for a Maxwellian the average energy $<\mathrm{Ek}>=(3 / 2) \mathrm{Tk}$, so that for $\mathrm{U}^{235}$ the average energy of the six delayed neutrons spectra varies from about $0.4 \mathrm{MeV}$ ( $\mathrm{T} 1=0.27$ $\mathrm{MeV}$ ) to about $0.55 \mathrm{MeV}(\mathrm{T} 4=0.371 \mathrm{MeV})$.

To completely describe the time and energy distribution of delayed neutrons with the model used here all we need are 18 parameters for each isotope: six Pk, six $\lambda \mathrm{k}$, and six Tk; no complicated data bases are requires; just these simple 18 parameters per isotope. Since we assume the delayed neutrons are emitted from stationary fission products the emission will be isotropic in the Lab system and we do not need any extra data to describe the angular distribution. For example, for $\mathrm{U}^{235}$ the 18 parameters in the above table are all we need to describe the emission of delayed neutrons in energy, time and direction.

\section{Analytical Time Dependence}

First let's look at the analytical solution for the time dependence after a fission has occurred at time $\mathrm{t}=0$. The ENDF/B-VI models is, 
$P\left(E, E^{\prime}, t\right) d t d E^{\prime}=\left\langle v d(E)>\sum_{k=1}^{6} \mathrm{P}_{\mathrm{k}} * \lambda_{\mathrm{k}} * \operatorname{Exp}\left(-\lambda_{\mathrm{k}} * \mathrm{t}\right) \chi_{\mathrm{k}}\left(E^{\prime}\right) d t d E\right.$

We will first look at the time dependence integrated over final energy,

$$
P(E, t) d t=\left\langle v d(E)>\sum_{k=1}^{6} \mathrm{P}_{\mathrm{k}} * \lambda_{\mathrm{k}} * \operatorname{Exp}\left(-\lambda_{\mathrm{k}} * \mathrm{t}\right) d t\right.
$$

So that you can easily see the fraction of neutrons emitted, I will divide by the delayed fraction, and present both the differential and integral results,

$$
\begin{aligned}
& P(t) d t=\sum_{k=1}^{6} \mathrm{P}_{\mathrm{k}} * \lambda_{\mathrm{k}} * \operatorname{Exp}\left(-\lambda_{\mathrm{k}}{ }_{\mathrm{t}}\right) d t \quad \text { Differential }
\end{aligned}
$$

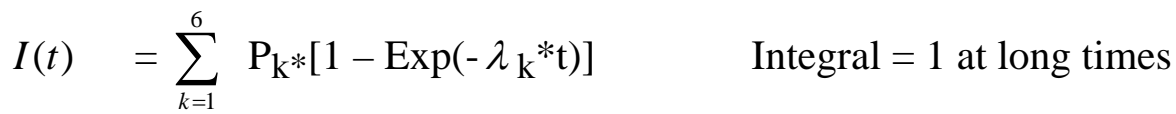

The below results for $\mathrm{U}^{235}$ show that after 100 seconds the differential emission has been reduced by a factor of roughly one thousand. To give you a feel for when the delayed neutrons are emitted, the integral results on the left figure below show that the percentage of delayed neutrons emitted by the following times: (0.01 seconds $0.5 \%)$; ( 0.1 seconds 4.3\%); ( 1 second 28\%); (10 seconds 77\%); (100 seconds 98\%); (430 seconds 99.99\%; not shown on the below figures which only extends up to 100 seconds).

The differential results on the right figure below show that by 100 seconds the delayed emission is exponentially decreasing according to a simple single exponential, i.e., on a linear-log scale the shape approaches a straight line, corresponding to the longest of the six time decay modes.
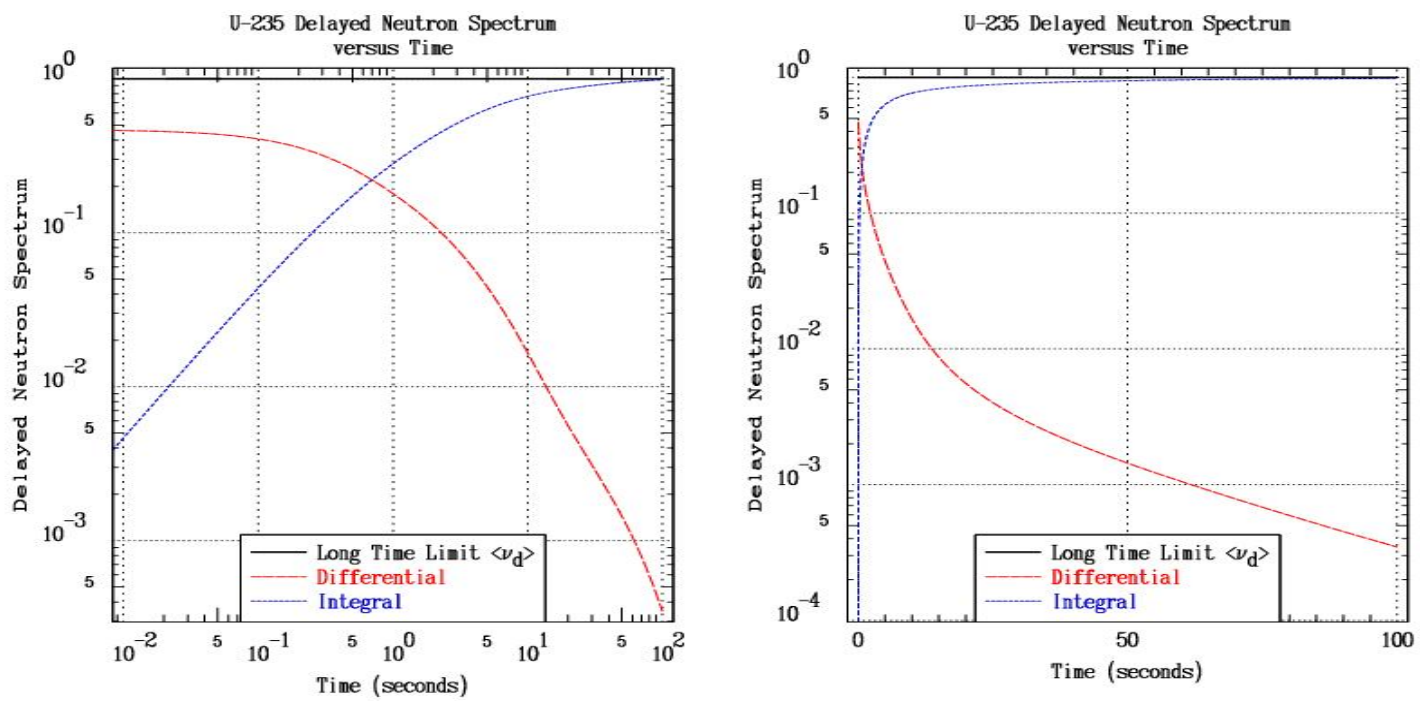


\section{Comparison of Analytical and TART Time Dependent Solutions}

Using a TART broomstick calculation we can obtain an absolute comparison between the analytical and TART solutions. The comparison will be absolute in terms of both magnitude and shape in time. Per source neutron the analytical solution for the time dependent leakage of delayed neutrons from the broomstick due to a monoenergetic neutron source at thermal energy $(0.0253 \mathrm{eV})$ is,

$<v d>\Sigma f / \Sigma t \sum_{k=1}^{6} \mathrm{P}_{\mathrm{k}} * \lambda_{\mathrm{k}} * \operatorname{Exp}\left[-\lambda_{\mathrm{k}} * \mathrm{t}\right]$

Using the above values for $\mathrm{U}^{235}$,

$0.01398 \sum_{k=1}^{6} \mathrm{P}_{\mathrm{k}} * \lambda_{\mathrm{k}} * \operatorname{Exp}\left[-\lambda_{\mathrm{k}} * \mathrm{t}\right]$

At $=0, \sum_{k=1}^{6} \mathrm{P}_{\mathrm{k}} * \lambda_{\mathrm{k}}=0.46856$

So that the solution will be the sum of the six exponentials absolutely normalized at $\mathrm{t}=0$ to 0.00655 . The below figure shows a comparison between the absolute analytical answer and a TART calculation for times up to 100 seconds ( $10^{8} \mu$ seconds) after fission. The left hand figure uses log-log scaling to show the excellent absolute agreement at short times. The right hand figure uses linear-log scaling to show the longer time comparison. If TART samples a million fissions we expect only 16,700 delayed neutrons. On the below figure results are distributed in 1000 time bins, which is the reason for the statistical noise for longer times. Overall I judge the agreement here to be excellent, verifying that TART is correctly sampling the time dependence of the delayed neutron emission.
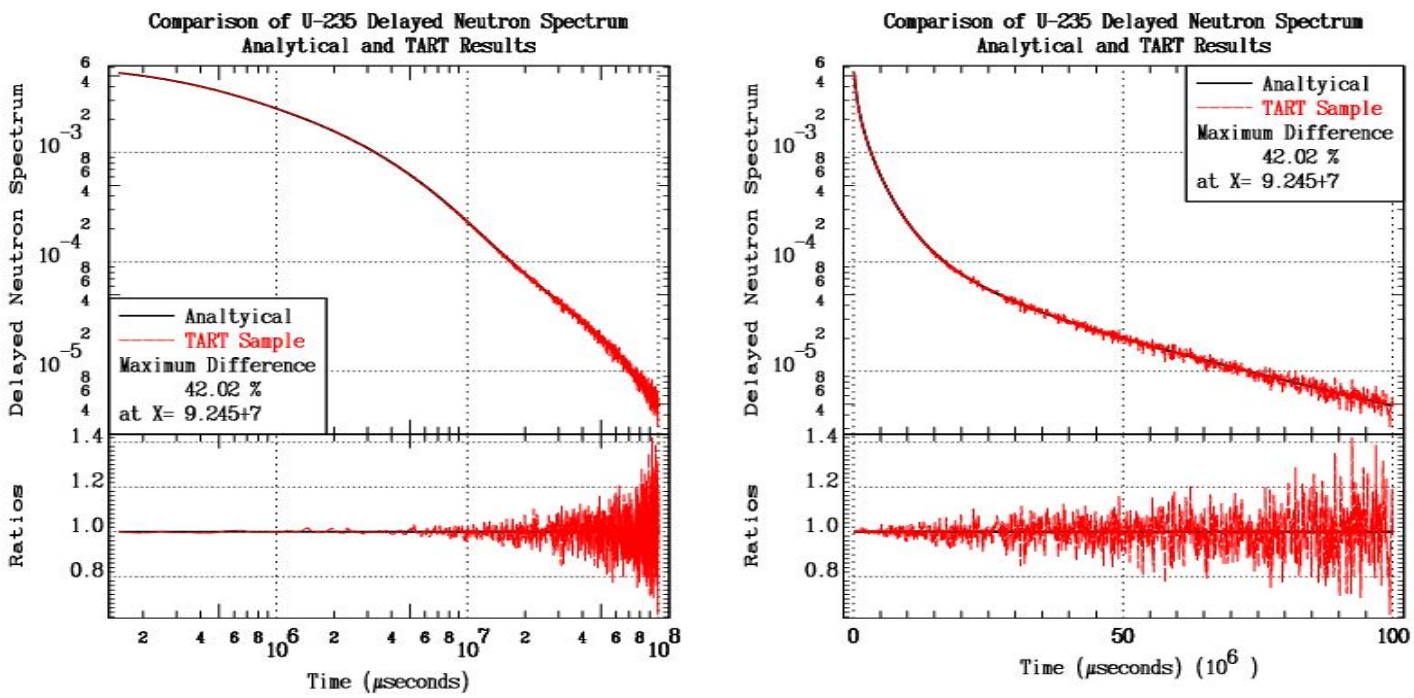


\section{TART Energy Dependent Solution}

The below figure shows energy dependent broomstick results for thermal neutrons incident on $\mathrm{U}^{235}$ for prompt times ( 0 to $4 \mu \mathrm{sec}$ ) ) and delayed times ( $4 \mu \mathrm{sec}$. to infinity). The prompt spectrum is composed of elastic scatter (the narrow peak around thermal energy broadened due to thermal motion) and prompt fission (the high energy neutrons). The delayed spectrum is composed only of delayed neutrons. Note that the prompt and delayed neutrons both have the same square root of E' low energy dependence. Note also the softer (lower energy) delayed spectrum relative to the prompt spectrum.

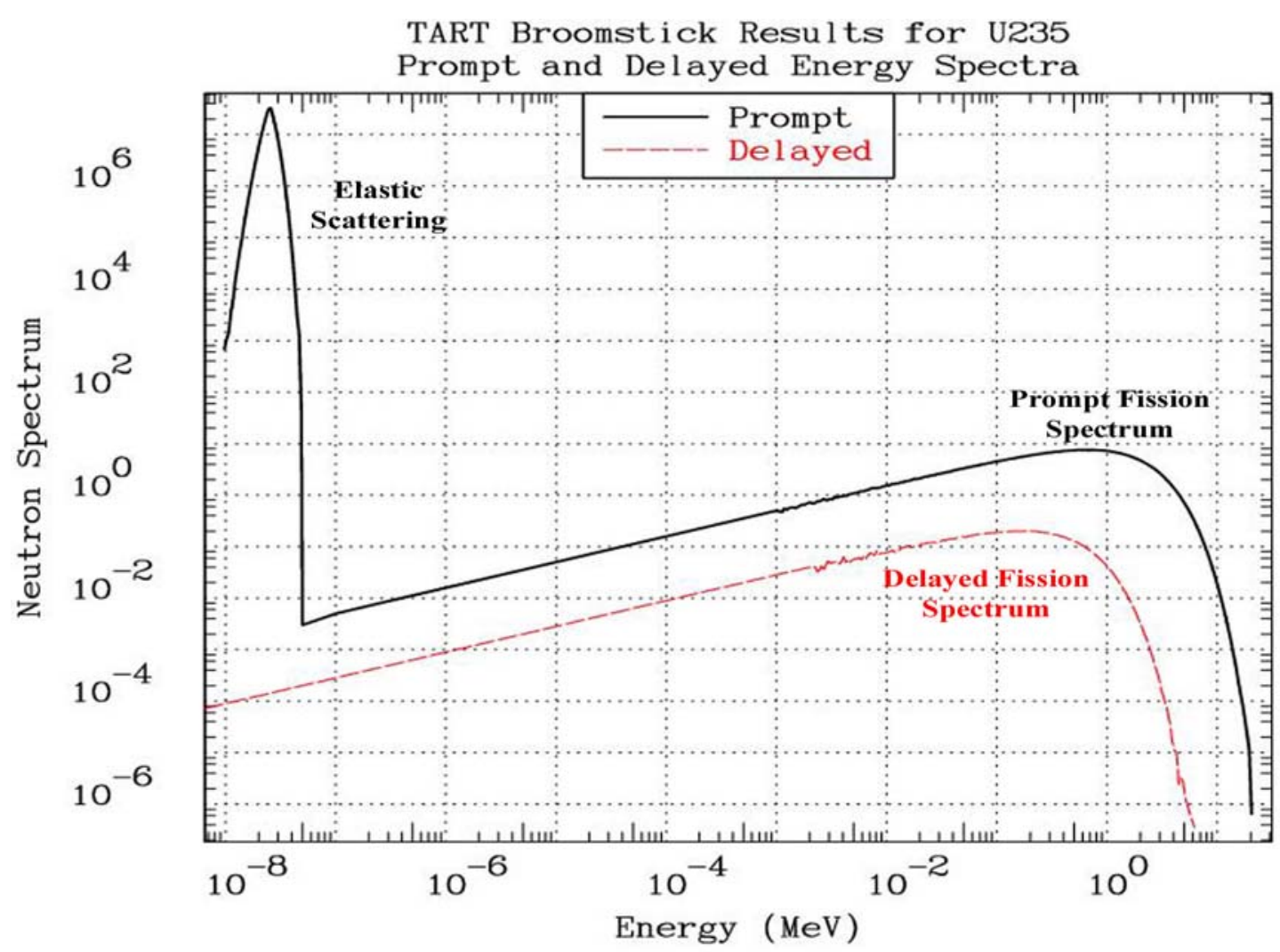

\section{Summary of Broomstick Results}

The above results illustrate time and energy dependent delayed neutron results as calculated by TART using a broomstick. In addition the broomstick calculation was also used to verify that the delayed emission is isotopic in the Lab system. The combination of broomstick results verifies that sampling delayed neutrons in time, energy and direction has been properly implemented in TART. 


\section{Steady State Solutions}

In any steady state, that is time independent problem, the assumption is that the system has existed in this state for all past times. In this case with the ENDF/B-VI model the fission products will have reached saturation, and the rate at which delayed neutrons are emitted exactly balances the rate at which fission creates the fission products. This means that the rate of emission of neutrons from fission at any time will be the prompt yield plus the delayed yield; in other words the total yield. This is why the normal assumption for steady state problems to use the total neutrons per fission is valid. Again, as a reminder this model implies that there is no significant fission product burn-up, e.g., if there is any significant fission product burn-up it will reduce the number of delayed neutrons that are actually emitted, in which case the normal assumption to use total neutrons per fission is incorrect.

In steady state problems, the delay time of delayed neutrons plays no role, but the difference between the prompt and delayed emission energy spectra can be important. The largest effect that I can think of would be in a system where $U^{238}$ fast fission makes an important contribution to K-eff. In this case, compared to modeling all neutrons emitted in the prompt emission energy spectrum, modeling the delayed neutrons in the softer delayed emission energy spectra moves the delayed fraction of neutrons below the effective $\mathrm{U}^{238}$ fission threshold, thereby reducing K-eff. How important this effect is depends on how important the $\mathrm{U}^{238}$ contribution is to K-eff.

\section{Minimum Enriched Uranium}

The most extreme case that I can think of is the theoretical problem involving only pure uranium and calculating the minimum per-cent of $\mathrm{U}^{235}$ in uranium in an infinite system that can be made critical. Here $\mathrm{U}^{238}$ fast fission plays a crucial role, and the results illustrate the important effect of properly modeling the delayed neutrons emission spectra. Note that the minimum per-cent here based upon only uranium, is not the same as the minimum per-cent in a general case if we include other materials, such as moderators. In the general case this per-cent is less than $1 \% \mathrm{U}^{235}$. Here I only consider pure uranium in order to maximize the effect of the delayed neutron spectrum. Later we will see that there can even be a somewhat smaller but important effect on thermal systems.

Using TART to first model this pure uranium problem with all fission neutrons (prompt and delayed) emitted in the prompt energy spectrum I find that the minimum for K-eff = 1.000 is $6.08 \% \mathrm{U}^{235}$. Repeating this calculation with delayed neutrons in the delayed emission spectrum we find that with the same per-cent $\mathrm{U}^{235}$ K-eff $=0.9978$, i.e., a

reduction of $0.22 \%$. In order to make this system critical I had to increase the $\mathrm{U}^{235}$ from $6.08 \%$ to $6.13 \%$, an increase of not quite $1 \%$. These differences may seem small, but compare this to the results of a recent study on how accurately we can calculate K-eff for 
thermal systems [8] which found today a variety of Monte Carlo codes can all calculate K-eff within roughly $0.1 \%$. Compared to the $0.1 \%$ accuracy we can reproduce today, the $0.22 \%$ difference due to correctly modeling delayed neutron emission is large. Below I show a comparison of the energy dependent neutron production emitting all neutrons in the prompt energy spectra or total using prompt and delayed energy spectra. In this case we see a shift of the total to lower energy due to the softer delayed neutrons. Note the "bump" in the production near $2 \mathrm{MeV}$, which is characteristic of fast fission due to $\mathrm{U}^{238}$.

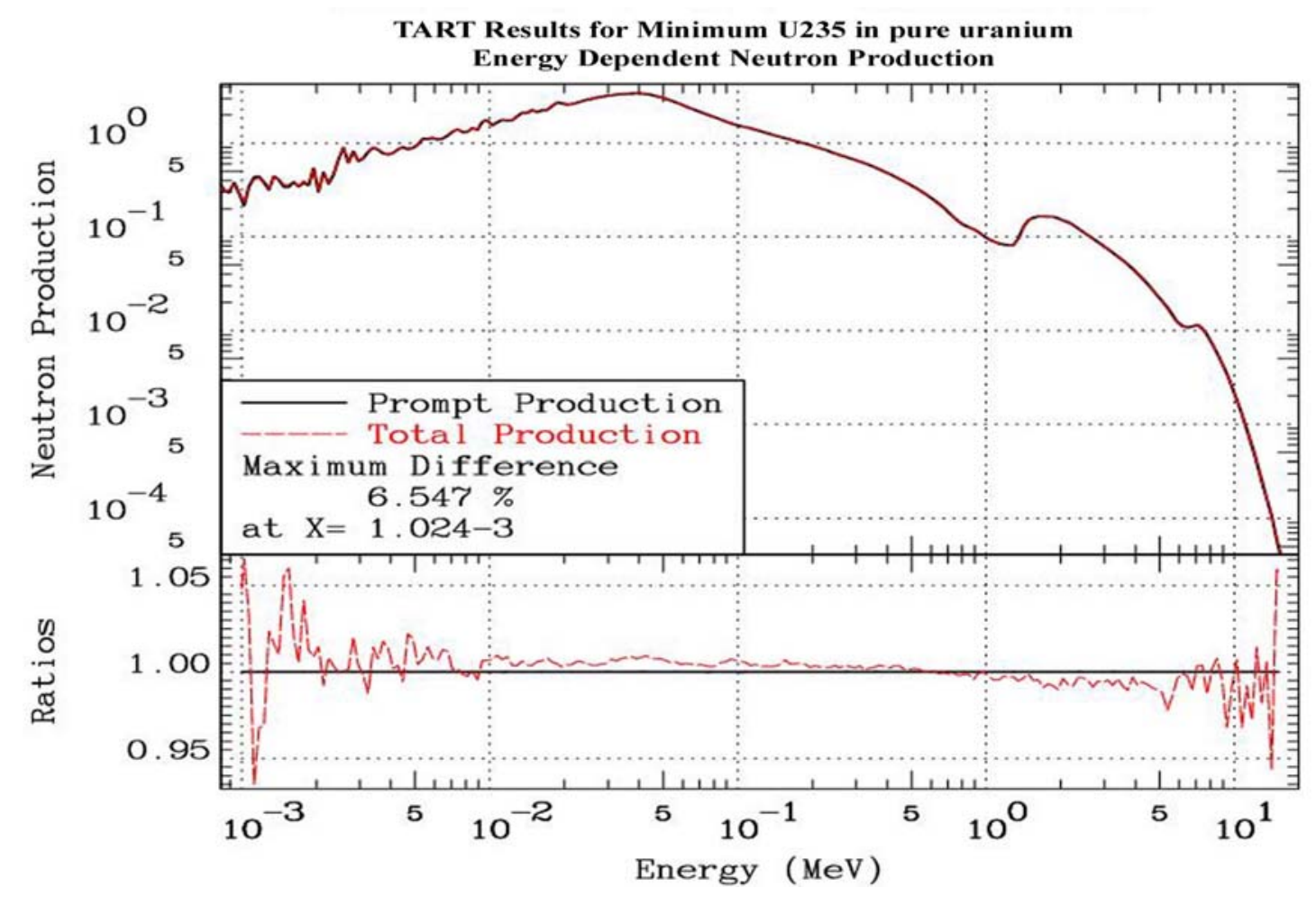

\section{Godiva: Highly Enriched Uranium}

To illustrate the opposite extreme I next consider a case with highly enriched uranium, i.e., very little $\mathrm{U}^{238}$. The next figure shows the energy dependent neutron production in Godiva (a bare sphere of highly enriched uranium). The curve labeled "prompt" corresponds to emitting all neutrons using the prompt emission energy spectrum. The curve labeled "total" corresponds to emitting the neutrons using the prompt and delayed emission energy spectra. We can clearly see the difference due to the softer delayed neutrons. In this case the net effect on K-eff is quite small (prompt 1.0017, delayed 1.0018). Here we see the shift in energy of the neutron production, but this has little net effect on K-eff because $\mathrm{U}^{238}$ makes little contribution to K-eff and neutrons in the prompt or delayed emission spectra make similar contributions to K-eff based on $\mathrm{U}^{235}$ fission. Compare the above figure for the minimum $\mathrm{U}^{235}$ to the below figure, and note that above we see the "bump" due to $\mathrm{U}^{235}$ fast fission, whereas below there is no "bump" 
because there is little $\mathrm{U}^{238}$ in this problem. On both the above and below figures you can see a smaller "bump" at around $6 \mathrm{MeV}$, which is due to $(n, 2 \mathrm{n})$.

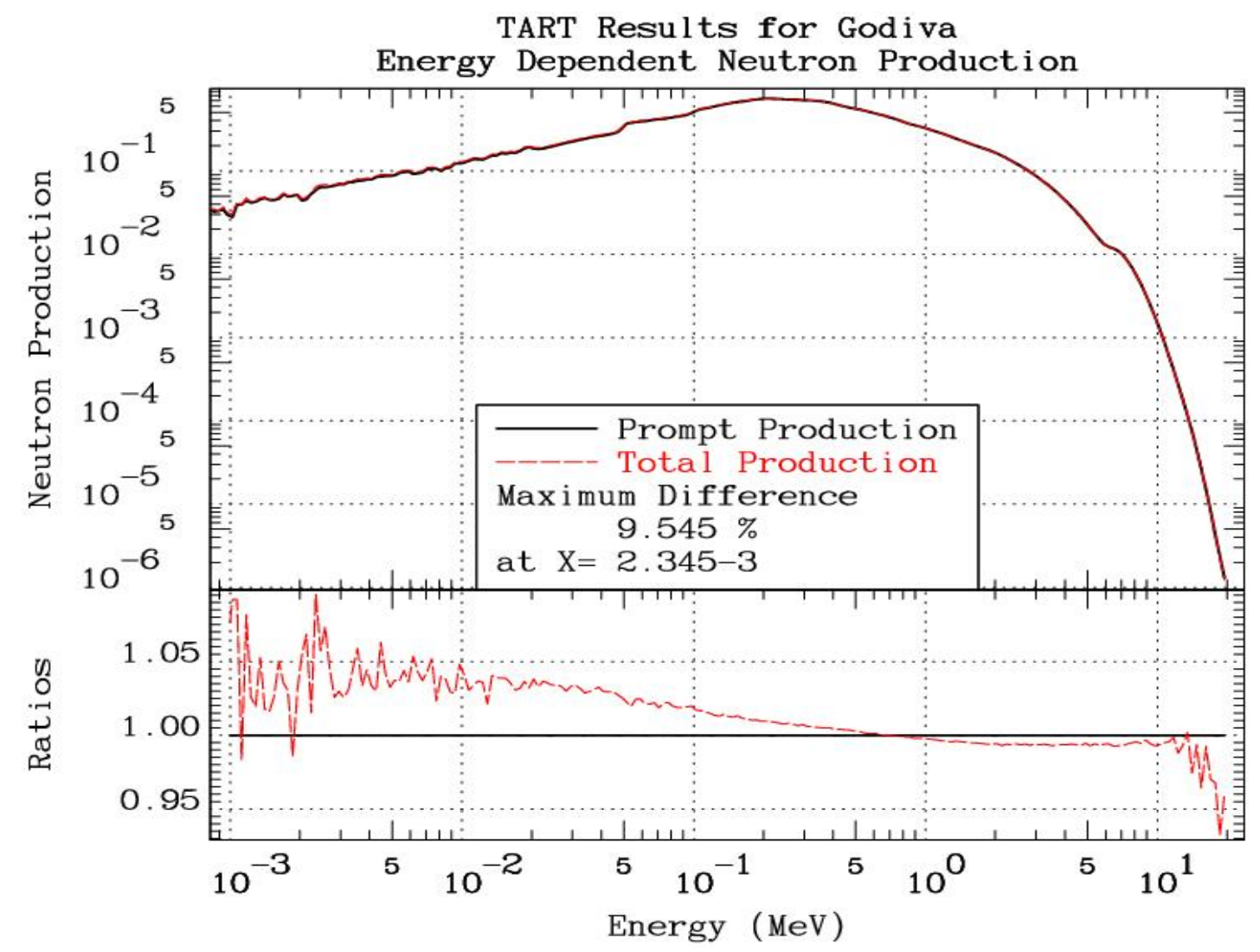

What these results for low and high enriched uranium show is that emitting delayed neutrons in the softer delayed emission spectrum rather than the prompt emission spectrum may or may not be important for simple integral parameters such as K-eff. In both cases we can see differences in the energy dependent neutron production and there is no significant difference in running time using one model or the other, so that it seems worthwhile to always use the physically better model of delayed neutrons emitted in the softer delayed neutron emission spectrum. 


\section{A Quick Survey of Many Fast Critical Assemblies}

The TART code system is distributed with TART input decks for over 500 critical assemblies. This includes a set of 68 fast neutron assemblies, including $\mathrm{U}^{233}, \mathrm{U}^{235}$, or $\mathrm{Pu}^{239}$ as fuel, and reflecting materials throughout the periodic table. As such this is an excellent set to use to obtain a quick overview of the magnitude of the effect of delayed neutrons.

I ran all 68 problems with all neutrons emitted in the prompt fission emission spectrum. I repeated the calculation with delayed neutrons emitted in the delayed fission emission spectrum. The table below summarizes the results. Diff \% is here defined as,

100\%(Prompt - Delayed)/Delayed

So that a positive Diff \% means the Prompt is larger than the Delayed. We see small positive and negative effects of properly treating the delayed neutrons; up to $0.06 \%$. The net effect averaged over the 68 cases is that the Prompt results are slightly larger than the Delayed results, as shown by a net increase in K-eff of less than $0.02 \%$.

Note that the average value for all 68 assemblies for Prompt (0.99927) is slightly closer to unity that the Delayed results (0.99911). Please do not make mistake of assuming that this indicates that the physical model I used for the Prompt calculations is better than physical model I used for the Delayed calculations. Indeed we know that exactly the opposite is true. Often critical assemblies such as these are used in an attempt to "improve" nuclear data. This is a classic example of the importance of using the "best" possible physical model when attempting such "improvements".

Above I discussed the effect of emitting delayed neutrons in a softer spectrum to decrease K-eff by moving neutrons below the $\mathrm{U}^{238}$ effective fission threshold. From the table below we can see that in general the effect is not that simple. Moving neutrons to lower energy can reduce leakage and decrease the chance of absorption during slowing down, resulting in an increase in K-eff. Below we can see examples of both increasing and decreasing K-eff due to the combination of materials and geometry.

My conclusion is that the effect of delayed neutrons is not easily predictable, so that to be safe it is better to always include the best physical model by including a proper treatment of delayed neutrons.

\begin{tabular}{|c|c|c|c|c|c|c|}
\hline $\begin{array}{l}\text { Crit. } \\
\text { ID. } \\
======\end{array}$ & Fuel & Reflector & & $\begin{array}{l}\text { K-eff } \\
\text { Prompt } \\
========\end{array}$ & $\begin{array}{l}\text { K-eff } \\
\text { Delayed } \\
========\end{array}$ & $\begin{array}{r}\text { Diff. \% } \\
\text { ==ニ=ニ==ニ }\end{array}$ \\
\hline c10100 & $\mathrm{pu}-\mathrm{a}$ & be & 5.222 & $1.00 \odot 75 \odot \odot$ & 1..$\odot \odot 83 \odot \odot$ & $-\odot . \odot \odot 8 \%$ \\
\hline C20100 & $\mathrm{pu}-\mathrm{a}$ & be & 8.170 & $1.0 \odot 24 \odot \odot \odot$ & $1 . \odot \odot 2230 \odot$ & 0.017 \\
\hline c30100 & $\mathrm{pu}-\mathrm{a}$ & be & 13.000 & 1.0017300 & 1. $001690 \odot$ & $\odot .0 \odot 4$ \\
\hline c40100 & $p u-d$ & bare & & $\odot .9992200$ & $\odot .9993800$ & $-0.016 \%$ \\
\hline c50100 & pu-d & be & 3.690 & $\odot .998900 \odot$ & $\odot .9990400$ & -0.014 \\
\hline c60100 & $\mathrm{pu}-\mathrm{d}$ & be & 5.250 & 1.0000100 & 1.0000400 & -0.003 \\
\hline & pu-d & c & 3.830 & 1.0014 & 1. $0 \odot 1520 \odot$ & $-\odot .0 \odot 8$ \\
\hline c80100 & pu-d & $\mathrm{ti}$ & 8.000 & $\odot .9940100$ & $\odot .9942800$ & $-\odot .027$ \\
\hline
\end{tabular}




\begin{tabular}{|c|c|c|c|c|c|c|c|}
\hline c90100 & $p u-d$ & W & 4.700 & 1.0029800 & 1.0029400 & 0.004 & $\%$ \\
\hline c10010 & pu-d & $u-235$ & 0.660 & 1.0002600 & 1.0003200 & -0.006 & $\%$ \\
\hline c11010 & $p u-d$ & $u-238$ & 1.930 & ๑. 9938900 & $\odot .9940400$ & -0.015 & $\%$ \\
\hline c12010 & $p u-d$ & $u-238$ & 6.740 & ๑. 9979100 & ๑. 9981100 & -0.020 & $\%$ \\
\hline c13010 & $p u-d$ & $\mathrm{u}$ & 4.130 & 1.0008500 & 1.0009900 & -0.014 & $\%$ \\
\hline c14010 & $p u-d$ & $\mathrm{u}$ & 19.600 & ๑. 9980000 & ๑. 9985500 & -0.055 & $\%$ \\
\hline c10100 & $u-233$ & bare & & ๑. 9926100 & ๑. 9926100 & $\odot .000$ & $\%$ \\
\hline c20100 & $u-233$ & be & 2.050 & ๑. 9962200 & ๑. 9956900 & 0.053 & $\%$ \\
\hline c30100 & $u-233$ & be & 4.200 & ๑. 9989300 & ๑. 9987200 & 0.021 & $\%$ \\
\hline c40100 & $u-233$ & W & 2.440 & ๑. 9999800 & ๑. 9997900 & 0.019 & $\%$ \\
\hline c50100 & $u-233$ & W & 5.790 & 1.0015700 & 1.0012100 & 0.036 & $\%$ \\
\hline c60100 & $u-233$ & $u-235$ & 1.210 & ๑. 9978500 & $\odot .9978500$ & 0.000 & $\%$ \\
\hline c70100 & $u-233$ & $u-235$ & 1.980 & 1.0021300 & 1.0022100 & -0.008 & $\%$ \\
\hline c80100 & $u-233$ & $u-235$ & 4.820 & 1.0081800 & 1.0078500 & 0.033 & $\%$ \\
\hline c90100 & $u-233$ & $\mathrm{u}$ & 2.300 & ๑. 9978600 & $\odot .9975400$ & 0.032 & $\%$ \\
\hline c10010 & $u-233$ & $\mathrm{u}$ & 5.310 & 1.0002000 & ๑. 9998800 & 0.032 & $\%$ \\
\hline c11010 & $u-233$ & $\mathrm{u}$ & 19.910 & ๑. 9978300 & ๑. 9980100 & -0.018 & $\%$ \\
\hline c०01 & $u-235$ & be & 1.27 & ๑. 9921400 & $\odot .9921200$ & 0.002 & $\%$ \\
\hline $\mathrm{c} \odot \odot 2$ & $u-235$ & be & 2.54 & ๑.9940300 & $\odot .9935200$ & 0.051 & $\%$ \\
\hline $\mathrm{c} \odot 03$ & $u-235$ & $\mathrm{C}$ & 1.27 & 1.0013000 & 1.0012600 & 0.004 & $\%$ \\
\hline $\mathrm{c} \odot \odot 4$ & $u-235$ & $\mathrm{C}$ & 2.54 & ๑. 9962900 & ๑. 9960300 & 0.026 & $\%$ \\
\hline c०05 & $u-235$ & $\mathrm{mg}$ & 1.27 & ๑. 9930300 & ๑. 9925700 & 0.046 & $\%$ \\
\hline c००6 & $u-235$ & $\mathrm{mg}$ & 2.54 & ๑. 9983300 & ๑. 9978600 & 0.047 & $\%$ \\
\hline $\mathrm{c} \odot \odot 7$ & $u-235$ & al & 1.27 & ๑. 9938300 & ๑. 9936500 & 0.018 & $\%$ \\
\hline c००8 & $u-235$ & al & 2.54 & ๑. 9960300 & ๑. 9956200 & 0.041 & $\%$ \\
\hline c००9 & $u-235$ & $\mathrm{ti}$ & 1.27 & ๑. 9924700 & ๑. 9921200 & 0.035 & $\%$ \\
\hline c०1० & $u-235$ & $\mathrm{ti}$ & 2.54 & ๑. 9943100 & $\odot .9944300$ & -0.012 & $\%$ \\
\hline c011 & $u-235$ & $\mathrm{fe}$ & 1.27 & 1.0001200 & 1.0001100 & 0.001 & $\%$ \\
\hline c012 & $u-235$ & $\mathrm{fe}$ & 2.54 & ๑.9923800 & $\odot .9921700$ & 0.021 & $\%$ \\
\hline c013 & $u-235$ & ni & 1.27 & ๑. 9965900 & $\odot .9963200$ & 0.027 & $\%$ \\
\hline c014 & $u-235$ & ni & 2.54 & ๑. 9956200 & $\odot .9953300$ & ๑. 029 & $\%$ \\
\hline c०15 & $u-235$ & $\mathrm{cu}$ & 1.27 & ๑. 9921000 & $\odot .9920000$ & 0.010 & $\%$ \\
\hline c016 & $u-235$ & $\mathrm{cu}$ & 2.54 & ๑. 9983300 & $\odot .9979600$ & 0.037 & $\%$ \\
\hline c०17 & $u-235$ & mo & 1.27 & ๑. 9978500 & $\odot .9974900$ & 0.036 & $\%$ \\
\hline c018 & $u-235$ & mo & 2.54 & 1.0038200 & 1.0035900 & 0.023 & $\%$ \\
\hline c०19 & $u-235$ & mo-alloy & & 1.0015500 & 1.0011800 & 0.037 & $\%$ \\
\hline $\mathrm{c} \odot 20$ & $u-235$ & w & 1.27 & ๑. 9921200 & $\odot .9920500$ & $\odot .0 \odot 7$ & $\%$ \\
\hline c021 & $u-235$ & W & 2.54 & ๑. 9952000 & ๑. 9949900 & 0.021 & $\%$ \\
\hline c10100 & $u-235$ & bare & & ๑. 9994100 & $\odot .9992800$ & $\odot .013$ & $\%$ \\
\hline c20100 & $u-235$ & bare & & 1.0049000 & 1.0049600 & -0.006 & $\%$ \\
\hline c30100 & $u-235$ & bare & & 1.0017100 & 1.0017700 & $-\odot .0 \odot 6$ & $\%$ \\
\hline c40100 & $u-235$ & be & 2.222 & $\odot .9984600$ & $\odot .9984300$ & $\odot .0 \odot 3$ & $\%$ \\
\hline c50100 & $u-235$ & be & 3.260 & $\odot .9997500$ & $\odot .9994300$ & $\odot .032$ & $\%$ \\
\hline c6010० & $u-235$ & be & 4.710 & 1.0029700 & 1.0027300 & $\odot .024$ & $\%$ \\
\hline c7010९ & $u-235$ & be & 5.440 & $1.0 \odot \odot \odot 3 \odot \odot$ & ๑. $999790 \odot$ & $\odot .024$ & $\%$ \\
\hline c80100 & $u-235$ & be & 9.270 & 1.0005700 & ๑. 9999900 & 0.058 & $\%$ \\
\hline c90100 & $u-235$ & be & 11.790 & ๑. 9992200 & ๑. 9986200 & $\odot .060$ & $\%$ \\
\hline c10010 & $u-235$ & $\mathrm{C}$ & 10.160 & 1.0003500 & 1.0002000 & 0.015 & $\%$ \\
\hline c11010 & $u-235$ & $\mathrm{C}$ & 15.240 & ๑. 9965500 & $\odot .9962200$ & 0.033 & $\%$ \\
\hline c12010 & $u-235$ & $\mathrm{ni}$ & 4.940 & 1.0060300 & 1.0056600 & 0.037 & $\%$ \\
\hline c13010 & $u-235$ & $\mathrm{cu}$ & 5.030 & 1.0029000 & 1.0025200 & 0.038 & $\%$ \\
\hline c14010 & $u-235$ & $\mathrm{cu}$ & 10.560 & 1.0082300 & 1.0081000 & 0.013 & $\%$ \\
\hline c15010 & $u-235$ & W & 5.080 & 1.0098400 & 1.0095600 & 0.028 & $\%$ \\
\hline c16010 & $u-235$ & W & 10.160 & 1.0100800 & 1.0098800 & $\odot .020$ & $\%$ \\
\hline c17010 & $u-235$ & $\mathrm{pb}$ & 8.990 & 1.0019800 & 1.0018000 & 0.018 & $\%$ \\
\hline c18010 & $u-235$ & $\mathrm{pb}$ & 17.220 & $\odot .9955500$ & $\odot .9950900$ & $\odot .046$ & $\%$ \\
\hline c19010 & $u-235$ & $\mathrm{u}$ & 1.760 & 1.0021000 & 1.0016500 & 0.045 & $\%$ \\
\hline c20010 & $u-235$ & $\mathrm{u}$ & 4.470 & 1.0057300 & 1.0055400 & ๑. . 019 & $\%$ \\
\hline c21010 & $u-235$ & $\mathrm{u}$ & 9.960 & 1.0024200 & 1.0024200 & $\odot .000$ & $\%$ \\
\hline c22010 & $u-235$ & $\mathrm{u}$ & 18.010 & ๑. $998410 \odot$ & $\odot .9983400$ & $\odot .0 \odot 7$ & $\%$ \\
\hline \multicolumn{8}{|c|}{ 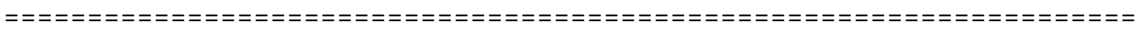 } \\
\hline Average & & & & $\odot .9992697$ & $\odot .9991128$ & $\odot .016$ & $\%$ \\
\hline
\end{tabular}




\section{Thermal Criticality Calculations}

In a recent study we examined how accurately today's neutron transport codes, particularly Monte Carlo codes, can calculate thermal systems [8]. The results were very encouraging since they demonstrated that today a variety of codes all calculate the same value for K-eff within roughly $0.1 \%$. The TART results in this report were based on modeling all fission neutrons as emitted prompt in the prompt neutron emission spectrum; I have no idea what the other codes used, since it was not defined in this study [8].

Using the delayed neutron model described here changes the TART pin-cell results by almost the $0.1 \%$ spread in code results seen in the earlier pin-cell study [8]. For example for the three free atom cases in this report the TART answers are: (1/2", prompt 1.0095, delayed 1.0088), (1/4”, prompt 1.0093, delayed 1.0091), (1/8”, prompt 1.0109, delayed 1.0109). Here "prompt" refers to the results with all neutrons emitted in the prompt energy emission spectrum, and "delayed" refers to the results with the delayed neutrons emitted in the delayed energy neutron spectrum. What these differences show is that the largest effect is seen for the $1 / 2$ " case, which contains $99.02 \% \mathrm{U}^{238}$ and only $0.98 \%$ $\mathrm{U}^{235}$, and roughly $10 \%$ of the neutron production occurs at high energy. In this case emitting the delayed neutrons in a softer energy spectrum moves the delayed neutrons below the effective $\mathrm{U}^{238}$ fission threshold decreasing K-eff by $0.07 \%$. The difference in K-eff is less in the $1 / 4$ " and $1 / 8$ " cases because here less of the neutron production occurs above the effective $\mathrm{U}^{238}$ fission threshold.

I will mention that the difference here is quite small, only $0.07 \%$ change in K-eff, but it is comparable to the difference seen between a variety of Monte Carlos codes and as such could effect the conclusions of this study [8]. The below figure shows the energy dependent neutron production for the $1 / 2$ " radius pin. Note the characteristic "bump" near $2 \mathrm{MeV}$ due to $\mathrm{U}^{238}$ fast fission, showing that even in this thermalized system $\mathrm{U}^{238}$ fast fission can play an important role.

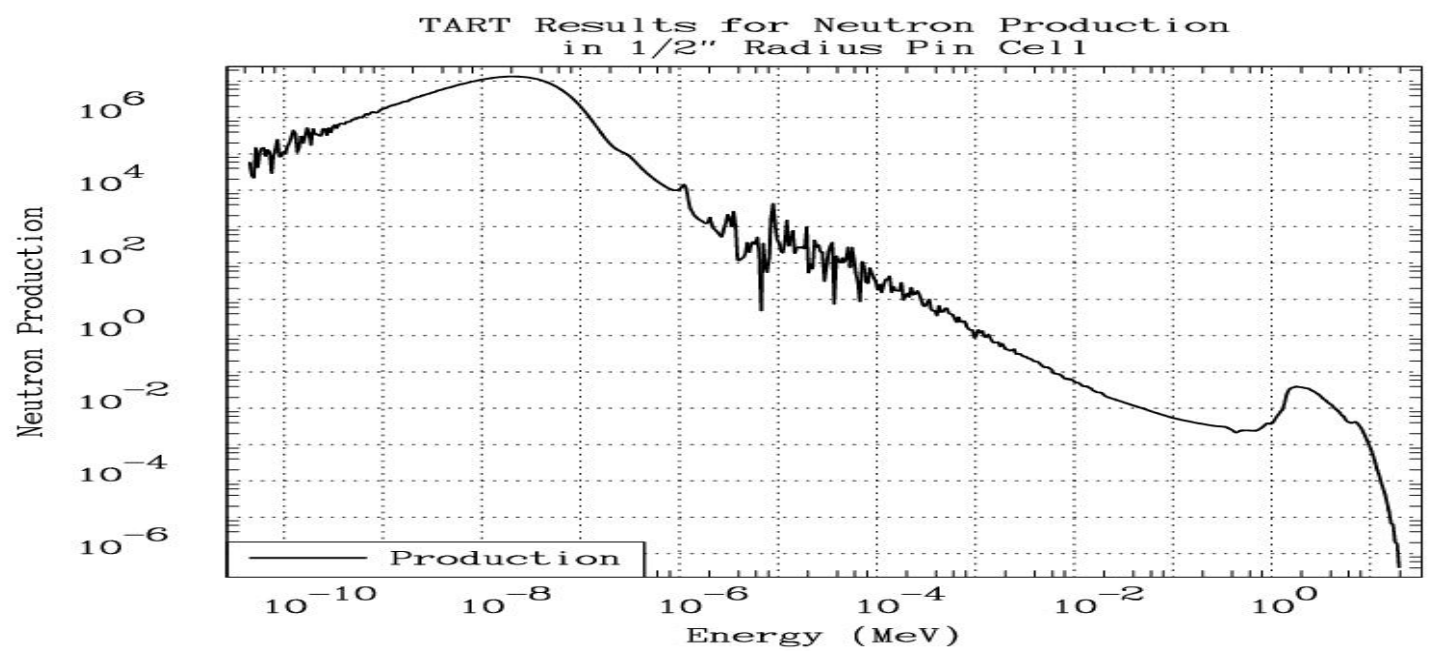




\section{Real Thermal Lattices}

LCT-06 [9] is a set of 18 measured, geometrically complex thermal assemblies. The below figures illustrates two of these assemblies (LCT06-1 and 18), which are both $19 \mathrm{x}$ 19 arrays of uranium oxide $\left(\mathrm{UO}_{2}\right)$ fuel, clad in aluminum, and surrounded by water; in these figures each color corresponds to a different spatial region for the TART model. The uranium is roughly $2.6 \% \mathrm{U}^{235}$, so that it is mostly $\mathrm{U}^{238}$. For the 18 assemblies the pitch and number of rods on each side were varied resulting in a range of hydrogen to uranium ratios.
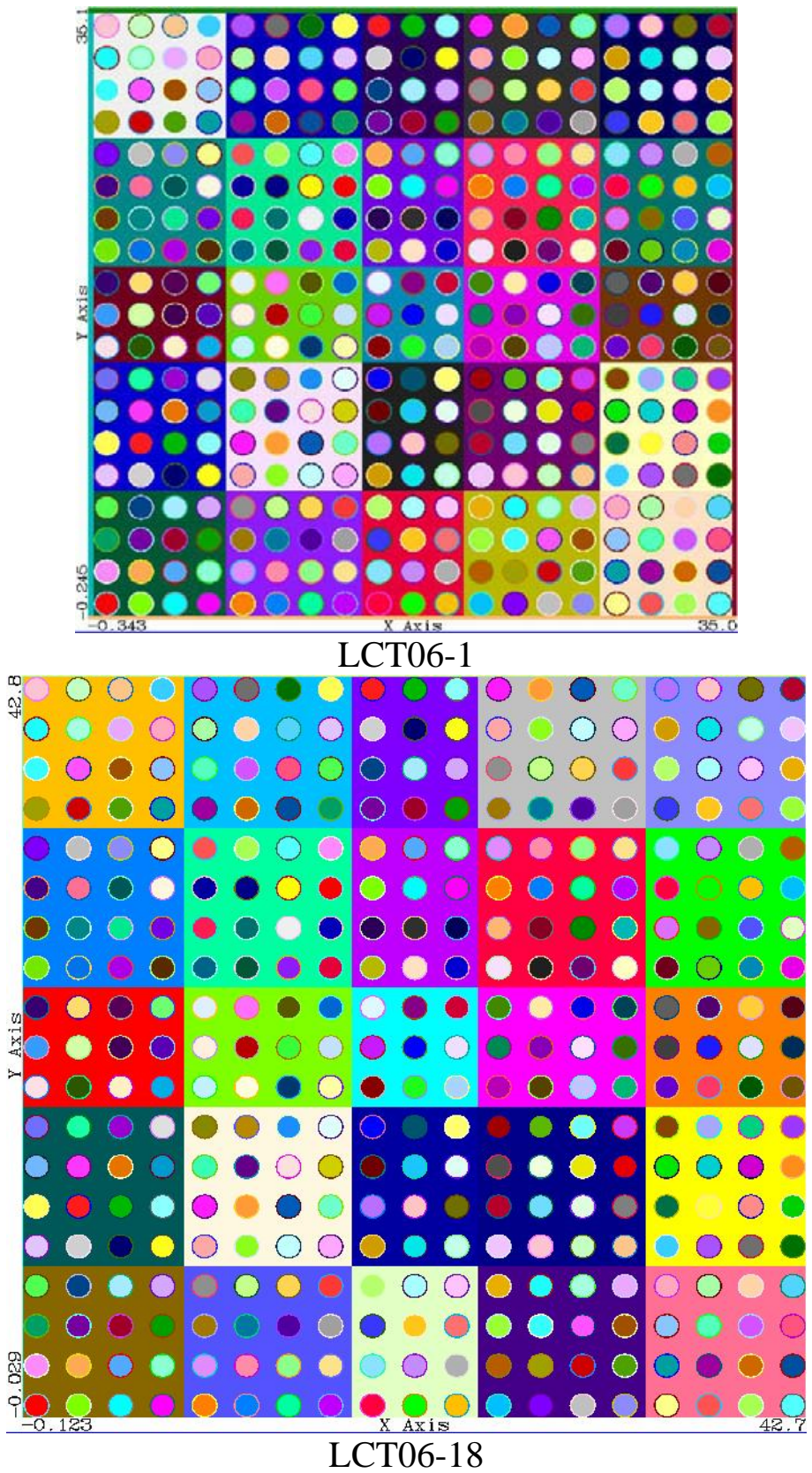
LCT06- 1 and 18 both are 19 by 19 arrays but have different pitches, with LCT06-1 being about $36.6 \mathrm{~cm}$ on a side and LCT06-18 about $42.7 \mathrm{~cm}$, resulting in H/U ratios of 4.33 and 8.65 , respectively. The table below defines the hydrogen to uranium ratio and number of rods on one side for each of the 18 assemblies.

Hydrogen to Uranium Ratio

\begin{tabular}{|l|c|c|c|c|c|c|c|}
\hline \multicolumn{2}{|c|}{ H/U 4.33 } & \multicolumn{2}{c|}{ H/U 5.28 } & \multicolumn{2}{c|}{ H/U 7.16 } & \multicolumn{2}{c|}{ H/U 8.65 } \\
\hline 1 & 19 rods & 4 & 17 rods & 9 & 16 rods & 14 & 15 rods \\
2 & 20 rods & 5 & 18 rods & 10 & 17 rods & 15 & 16 rods \\
3 & 21 rods & 6 & 19 rods & 11 & 18 rods & 16 & 17 rods \\
& & 7 & 20 rods & 12 & 19 rods & 17 & 18 rods \\
& & 8 & 21 rods & 13 & 20 rods & 18 & 19 rods \\
\hline
\end{tabular}

I have calculated these assemblies first emitting all fission neutrons in the prompt emission spectrum, and then repeated the calculation emitting delayed neutrons in the delayed neutron spectrum. These are preliminary TART 2004 results, so that the final results to be published with TART 2004 may vary slightly, but the difference between the results for the case of all prompt versus prompt and delayed will be consistent.

The below figure shows the energy dependent neutron production in LCT06-1; on the left is the differential production per $\mathrm{MeV}$, and on the right is the integral production normalized to unity integrated over all energies. Note the "bump" in the differential production near $2 \mathrm{MeV}$ which is characteristic of U238 fission. The integral production shows that even for this thermal system over $7 \%$ of the neutron production occurs above $1 \mathrm{MeV}$, due to U238 fast fission. Clearly the effect of U238 fast fission is important in this system.
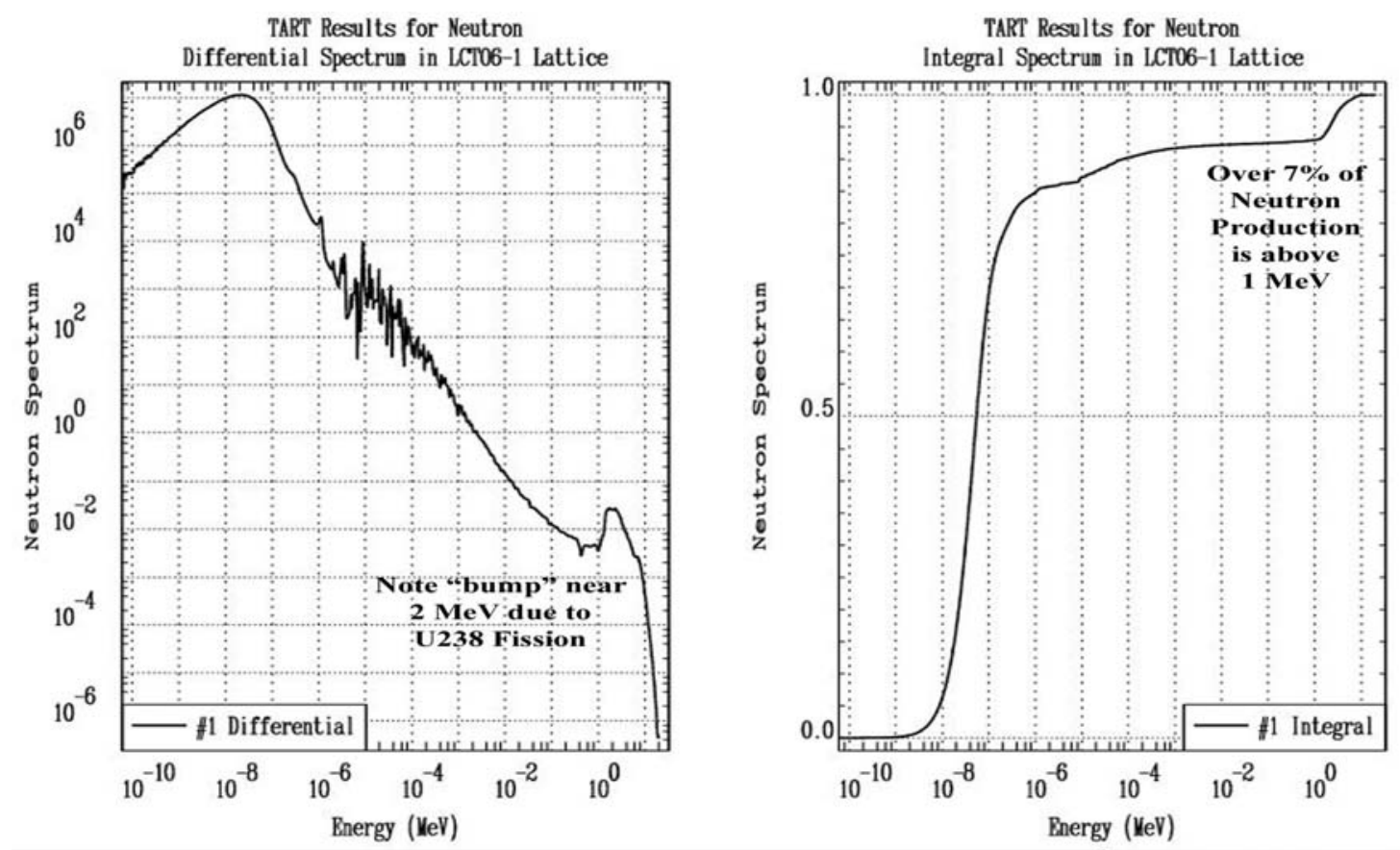
The below figure shows the energy dependent neutron production, absorption and leakage in LCT06-1; on the left is the differential results per $\mathrm{MeV}$ all normalized to one neutron produced, and on the right is the integral results each normalized to unity integrated over all energies. From here we can see that the production exceeds the absorption plus leakage from about 0.1 to $100 \mathrm{eV}$, as well as above $1 \mathrm{MeV}$. Note also that for this assembly a good deal of the leakage is at high energy, e.g., almost $40 \%$ is above 1 $\mathrm{MeV}$.
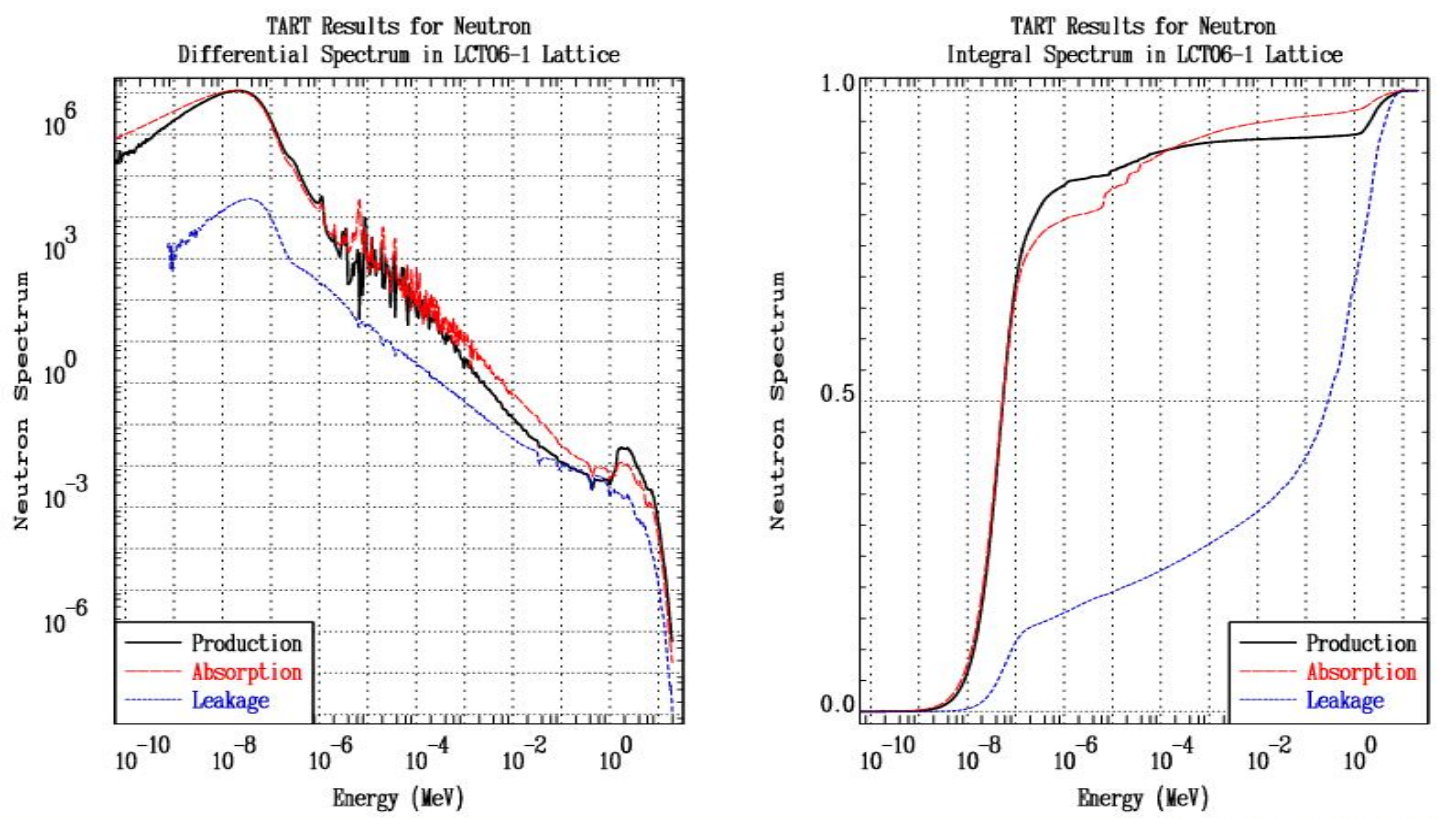
In this case proper treatment of delayed neutrons increases K-eff for all 18 assemblies. See the table below which compares results emitting all neutrons in the prompt fission energy spectrum (identified as Prompt) and emitting the delayed neutrons in the delayed fission energy spectrum (identified as Delayed). The per-cent difference in this table is,

100\%(Prompt - Delayed)/Prompt

In all 18 cases the difference is negative, indicating that K-eff in all cases increases when delayed neutron are treated properly. From the Prompt results we see is that as the H/U ratio increases K-eff increases from close to 1 for $\mathrm{H} / \mathrm{U} 4.33$ up to about 1.002 for $\mathrm{H} / \mathrm{U}$ 8.65, so that the trend we are seeing is a change in K-eff of only $0.2 \%$. This is comparable to the differences that we see between Prompt and Delayed results, which are up to about $0.1 \%$, and also increase with $\mathrm{H} / \mathrm{U}$ ratio. The net result is that if we were to try and use these assemblies to verify or improve evaluated data, clearly the effect of properly handling delayed neutrons is important and should be included in the analysis.

WARNING - WARNING - WARNING - the results in the table below are based on using a preliminary version of the TART 2004 nuclear data files - as such the absolute values of K-eff may vary for the final distributed TART 2004 package - only the change in K-eff due to properly using delayed neutrons should be considered important here.

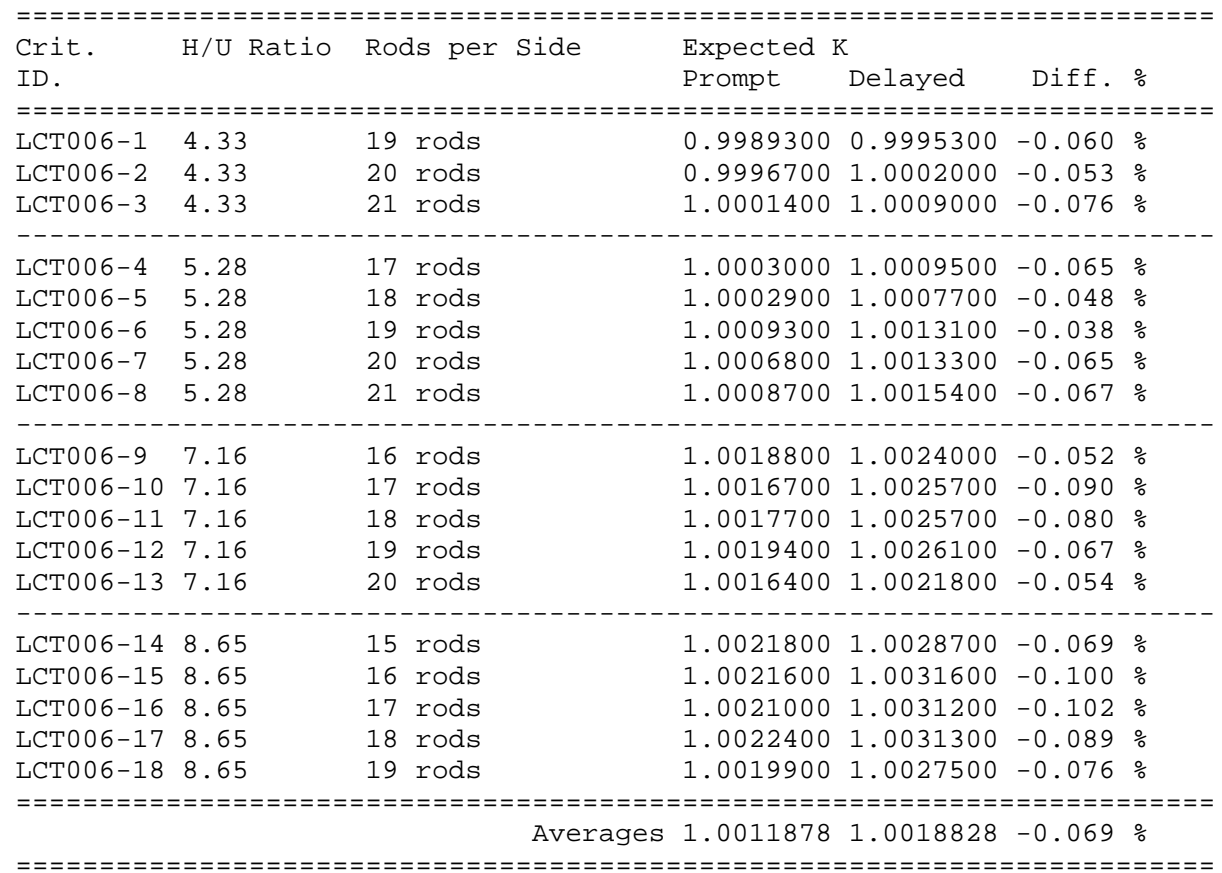


The below figure shows the energy dependent neutron production in LCT06-01 (H/U 4.33) and LCT01-18 (H/U 8.65). The figure on the left compares differential results where each is normalized to one neutron removed (absorbed or leaked). The figure on the right compares integral results where each is normalized to unity integrated over the entire secondary energy range. Comparing the two we can see the increasing slowing down power in LCT01-18, compared to LCT01-01, e.g., the slowing down production (and flux) is much lower, and the increase in the thermal spectrum below about $0.1 \mathrm{eV}$. Both of these effects contribute to the differences in K-eff that we see in the above table. Note that in both cases fast fission above $1 \mathrm{MeV}$ makes a considerable contribution to the production, but less for LCT06-18 (roughly 5\%) than LCT06-01 (roughly 7\%).
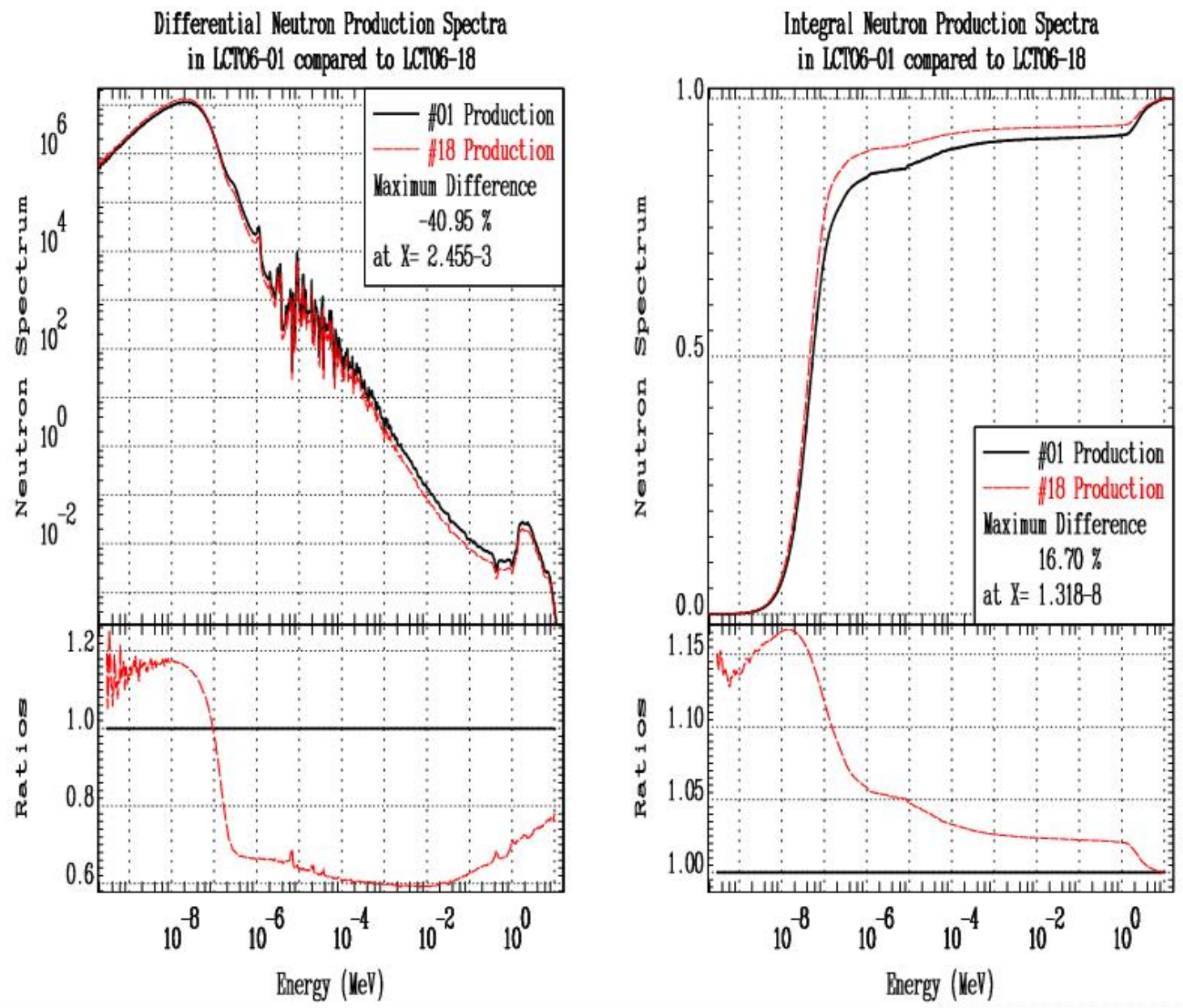


\section{Material Assay for Fissile Materials}

Delayed neutrons can be used to nondestructively interrogate a material in an attempt to determine if it contains fissile material. As an example, with TART I modeled a neutron fission spectrum incident on a sphere of pure $\mathrm{U}^{235}, 5 \mathrm{~cm}$ in radius, at a density of 18.7 grams/cc. I used two different time distributions for the source. One source was constant from 0 to 100 seconds and then zero for all longer times. The other source was more or less instantaneous; actually it was constant from 99.99 to 100.0 seconds, and then zero for all longer times. In each case exactly the same total number of neutrons was incident from the source. In each case I detected the time dependent leakage from the sphere.

The below figure shows a comparison of the results for these two sources. Because the total number of source neutrons incident was exactly the same in both cases the total number of delayed neutrons will also be the same. From the below figure we can see that after 100 seconds the strength of the constant source is lower, because roughly half of the delayed neutrons were emitted during the 100 seconds that the source was on and as such are masked by the much larger leakage of prompt sources during this time

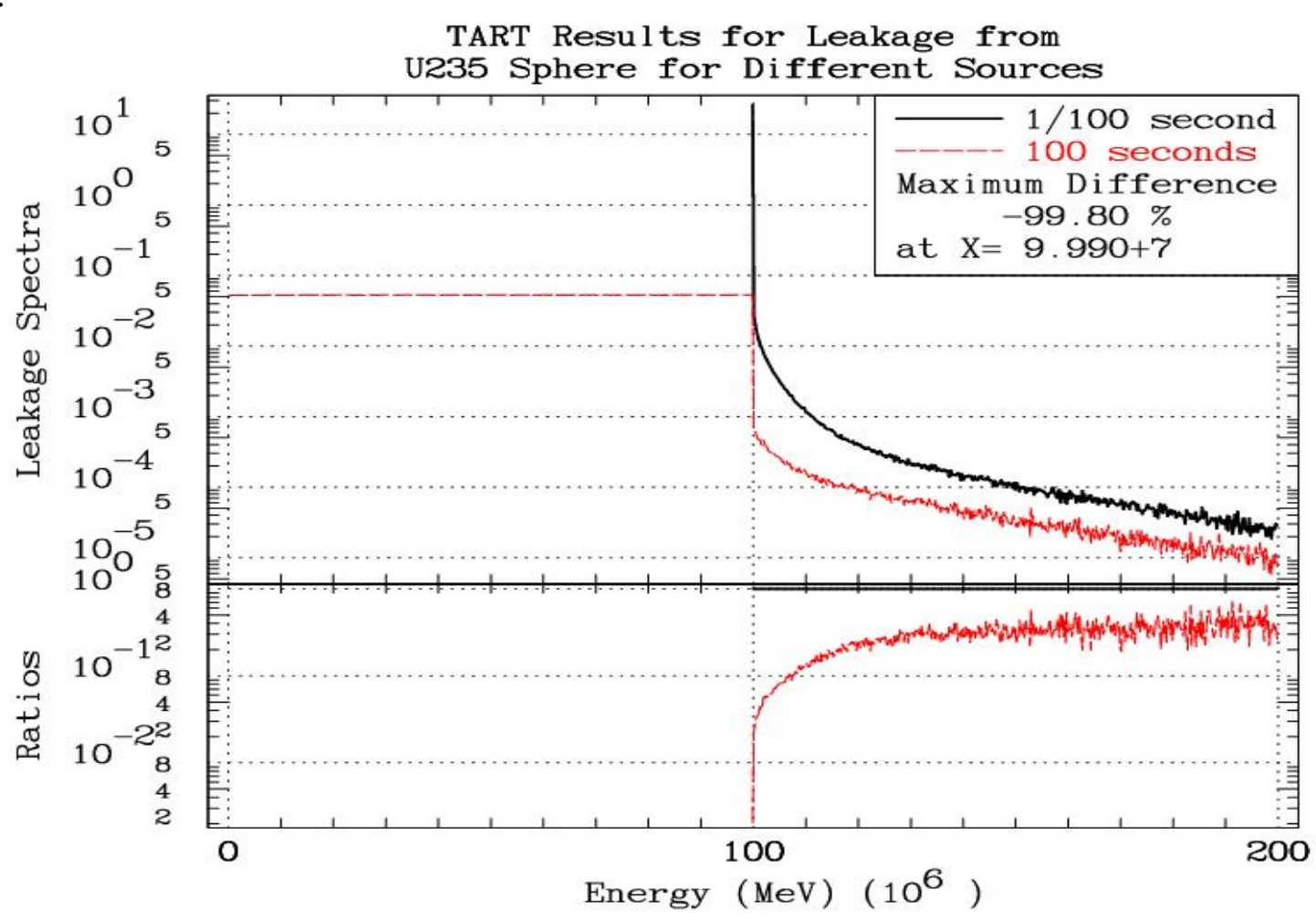




\section{Example of Spontaneous Fission}

For neutron source (not criticality) problems TART allows users to specify a spontaneous fission source for a few important isotopes, namely $\mathrm{Pu}^{240}, \mathrm{Cm}^{242}, \mathrm{Cm}^{244}$, or $\mathrm{Cf}^{252}$. These sources are often used to perform neutron correlation studies. For completeness delayed neutrons have now been included with the spontaneous sources.

For neutron correlation studies the correlated signal is due to a fission emitting all its fission neutrons at the same time. Properly treating the delayed neutrons as delayed in time means they are not longer emitted at the time as the fission and as such they are not part of the correlated signal, rather they are a background noise that can interfere with this signal.

With TART spontaneous sources may be used by themselves, e.g., calibrating to a $\mathrm{Cf}^{252}$ source in a non-multiplying medium. They can also be used in combination with other sources to simulate the total independent source in a medium, e.g., Pu240 spontaneous fission plus an external source incident on the medium to induce additional fissions. By tallying the results in binary files using ltype 12 (see the on-line TART input manual) the results for any number of sources may be combined.

Here I wish to merely demonstrate that delayed neutrons for spontaneous fission sources have been correctly implemented in TART. I do this using a complete imaginary situation where I turn on a spontaneous $\mathrm{Pu}^{240}$ source for 100 seconds, then turn it off and tally what happens out to 200 seconds. In this case I use a sphere of pure water (no induced fission) containing a point $\mathrm{Pu}^{240}$ source and I tally the time dependent leakage from the sphere. The below figure illustrates that TART is correctly emitting time delayed neutrons for the spontaneous source.

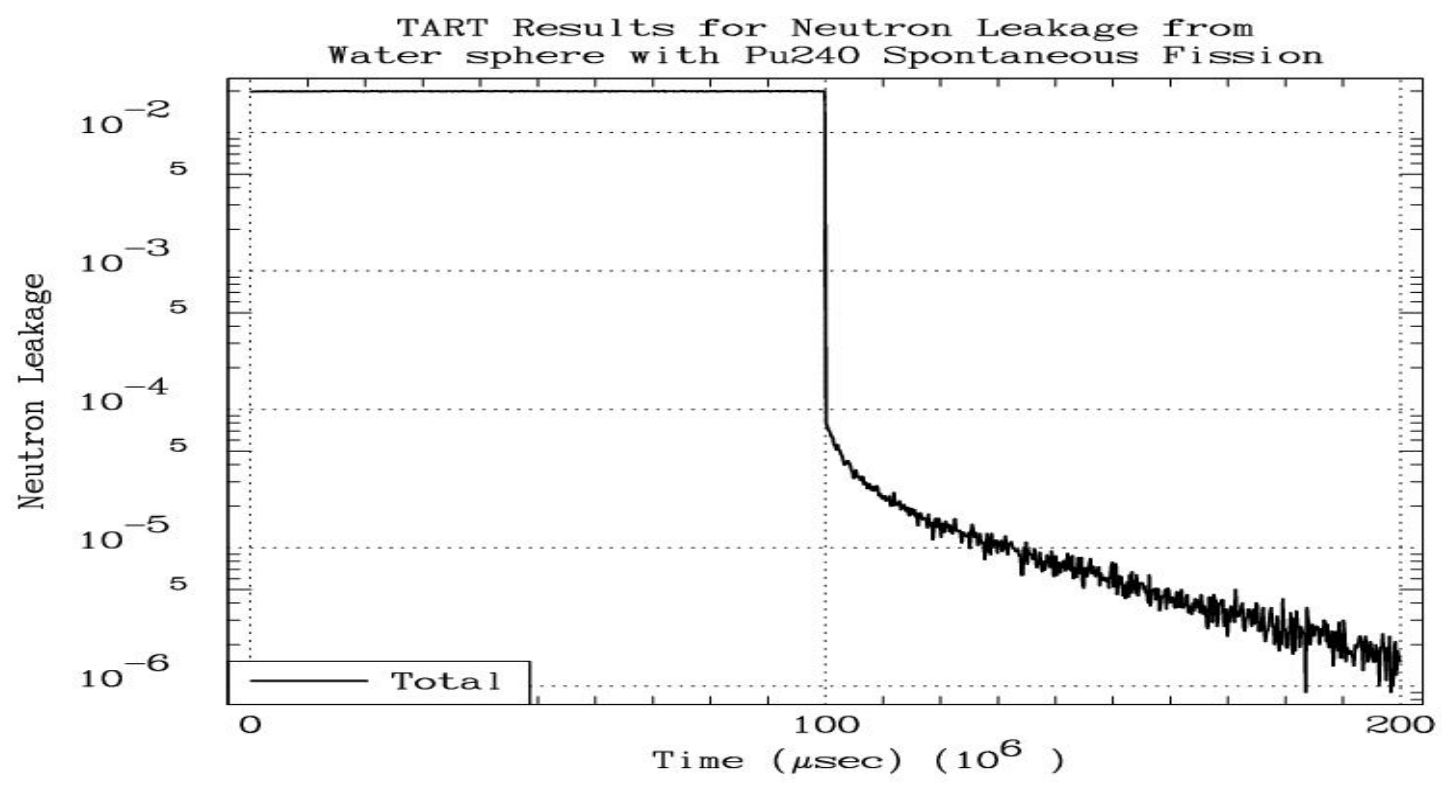




\section{Use Delayed Neutron Data with Care}

Care must be used with delayed neutron data when one uses fission product precursor densities to define the time dependent emission of delayed neutrons. The distribution of delayed neutrons emitted at any time, $t$, can be defined in terms of the decay rate of the fission product precursor densities as,

$\sum_{k} P_{\mathrm{k}} \lambda_{\mathrm{k}} C_{\mathrm{k}}(r, t) \chi_{\mathrm{k}}\left(E^{\prime}\right)$

The density of precursors is coupled to the neutron flux,

$\frac{\partial C k}{\partial t}=-\lambda_{\mathrm{k}} C_{\mathrm{k}}(r, t)+\int<v d k>\Sigma f^{*} N\left(r, E^{\prime}, t\right) d E^{\prime}$

In addition we need an initial condition defining the precursor densities at an initial time, say $\mathrm{t}=0, C_{\mathrm{k}}(r, 0)$.

The most frequent assumptions at the initial time are either that the precursor densities are zero, or have reached equilibrium due to a constant flux throughout the past,

$$
C_{\mathrm{k}}(r, 0)=\int<v d k>\Sigma f * N\left(r, E^{\prime}\right) d E^{\prime} / \lambda_{\mathrm{k}}
$$

The most common error in misusing the delayed neutron information is to assume that the initial precursor densities can be arbitrarily defined, whereas in fact we can see that these densities are directly coupled to the neutron flux by their production through neutron induced fission. Please be aware that any assumption concerning the initial precursor densities is directly equivalent to assuming an initial and past time flux. For example, if you assume that you know the initial values of the precursor densities and define them as constant, independent of position, you are assuming that the neutron flux is constant in space, which is usually a poor assumption. Be aware that the subsequent time dependent behavior of any system will depend - sometimes strongly - on the initial conditions that you define. So that using incorrect initial conditions may invalidate the result of your calculation.

This is why TART does not allow users to assume an initial precursor density. With TART if you want to "see" the time dependent effects of delayed neutrons you are forced to start with a flux and precursor densities that are zero before some initial time, and to allow the system to evolve forward in time to correctly define the time and spatial distribution of flux and delayed neutrons, and you can then introduce a perturbation to "see" the effect you are looking for, e.g., see the above example of material assay using a time dependent neutron source. From the above results you can see that after roughly 100 seconds systems will have evolved into a "true" spatial and time distribution of delayed 
neutrons characteristic of the system, so that subsequent time behavior will truly represent the system you are dealing with.

\section{Improved Delayed Neutron Data}

The ENDF/B-VI data file is now frozen [2], so that we cannot improve upon the current contents of this library. However, we can look forward to the creation of the new ENDF/B-VII library. The current delayed neutron data in ENDF/B-VI is a big step forward compared to earlier versions of ENDF/B that did not include any information to define the time and energy emission of delayed neutrons. In the hope that this excellent start with ENDF/B-VI leads to even further improvements in ENDF/B-VII here I make to few suggestions,

1) Delayed neutron information should be included for all fissile and fertile isotopes, and data should be added for important spontaneously fissioning isotopes. Personally I think the current uniform treating of delayed neutrons in ENDF/B-VI as defined by six precursor modes is an excellent idea; it allows a uniform treatment of delayed neutron emission in our application codes for all isotopes. Please do not interpret this to mean I am a fan of the six mode model; what I am a fan of is any uniform model that we can apply to all isotopes, be it six, eight, ten or whatever number of modes.

2) Coupling to specific fission product precursors or at least lumped precursors and defining burn-up cross sections would complete the equations defining the time dependence of the precursors. In most cases this is not an important effect, since we are talking about relatively short time periods, of the order of 100 seconds, and the flux would have to be enormous to cause significant burn-up during this time.

3) The energy spectra should be further improved, noting in particular the problems discussed here. The high energy variations now seems to be physically acceptable, but not the low energy histogram; hopefully this problem can be eliminated.

4) Photon emission due to delayed neutron emission should be added, since this can contribute to more delayed neutrons through $(\gamma, n)$ reactions in materials such as beryllium and deuterium, e.g., in heavy water reactors. The delayed photon signal could also be useful in material assay applications.

\section{Conclusion}

Here I have introduced a simple model of delayed neutron emission. The model uses the currently available ENDF/B-VI data [1, 2] to model the time dependence of the delayed neutron emission in six decay modes. The model uses a simplified model for the energy dependence that conserves the average energy of delayed neutrons emitted in the detailed ENDF/B-VI emission spectra; this is the only approximation to the currently available ENDF/B-VI data used in my simple model. 
This model has now been implemented in the TART [6, 7] Monte Carlo transport code. Results were presented to verify that this model has been correctly implemented in TART. In particular broomstick results were presented to verify the single event (collision) emission of delayed neutrons in time, energy, and direction. In addition results were presented to illustrate the effect of delayed neutrons on criticality. Lastly results were presented to illustrate how delayed neutrons can be used in material assay by using a neutron source incident on a given material; if fissile material is present the neutron source will induce fission and allow delaying neutrons to be detected.

The final point I would like to make is that implementing this model in a Monte Carlo code such as TART is trivial and does not add any appreciable overhead or running time to the code. Think about it: for each fission, only a fraction of $1 \%$ of the time is a delayed neutron emitted, which adds little complexity or running time. Since there are cases in which this effect is important it seems a shame not to improve the physics in our codes by including an accurate model of delayed neutron emission.

TART users should see the Appendix for guidance in using delayed neutrons in TART.

\section{Acknowledgement}

I thank Chris Clouse, LLNL, for reviewing a preliminary version of this paper, and making valuable suggestions and corrections to it; I feel that the final paper is significantly improved because of Chris' efforts. I also thank Maurice Greene, ORNL, for reviewing and suggesting improvements, particularly with regard to converting Cullenish to English. I also thank Bob MacFarlane, LANL, and Bob Little, LANL, for reviewing and suggesting improvements.

\section{References}

[1] "Data Formats and Procedures for the Evaluated Nuclear Data File, ENDF-6", ENDF102, Brookhaven National Laboratory, April 2001, ed. V. McLane, available on-line at http://www.osti.gov/bridge/product.biblio.jsp?osti_id=781830.

[2] The ENDF/B-VI data library is now frozen; the next change in the nuclear data will be in the new ENDF/B-VII data library. The archived ENDF/B-VI data is now available on-line as "POINT 2004: A Temperature Dependent ENDF/B-VI, Release 8 Cross Section Library", Lawrence Livermore National Laboratory, UCRL-TR-202284, April 2004, by D.E. Cullen, within the United States http://www3.nndc.bnl.gov/point2004/ as well as in Europe http://amdu1.iaea.org/point2004/

[3] "Physics of Nuclear Kinetics", by G. Robert Keepin, Los Alamos Scientific Laboratory, published by Addison-Wesley Publishing Company, Inc., 1965; this is the classic textbook on delayed neutrons.

[4] "Introduction to Nuclear Reactor Kinetics", by Daniel Rozon, Institut de genie

nucleaire, Ecole Polytechnique de Montreal, published in English by Polytechnic 
International Press, 1998; this is a more recent textbook that is an excellent complement to Keepin's classic on delayed neutrons.

[5] “The LLL Evaluated Nuclear Data Library (ENDL): Evaluation Techniques, Reaction Index, and Description of Individual Evaluations”, UCRL-50400, Vol. 15, Part A, Sept. 1975, Lawrence Livermore National Laboratory, by R. J. Howerton, et. al,

[6] “TART 2002: A Coupled Neutron-Photon 3-D, Combinatorial Geometry Time Dependent Monte Carlo Transport Code," Lawrence Livermore National Laboratory, UCRL-ID-126455, Rev. 4, November, 2002, by D.E. Cullen; documentation available on-line at http://www.llnl.gov/cullen1/mc.htm. This is the most recent public release of TART, but it does not include the delayed neutron model described here; see [7] below.

[7] "TART 2004: A Coupled Neutron-Photon 3-D, Combinatorial Geometry Time Dependent Monte Carlo Transport Code," to be published, by D.E. Cullen. The delayed neutron model described here is available in TART04-3 and all later versions of TART; regarding availability contact D.E.Cullen cullen1@llnl.gov.

[8] "How Accurately can we Calculate Thermal Systems?", with many authors, Lawrence Livermore National Laboratory, UCRL-TR-203892, April 2004, by D.E. Cullen, et al., available on-line at http://www.llnl.gov/cullen1/pin_cell.htm.

[9] “International Handbook of Evaluated Criticality Safety Benchmark Experiments”, editor Blair Briggs, NEA/NSC/DOC(95)03, September 2003 Edition.

\section{Appendix: Using Delayed Neutrons in TART}

This section is intended strictly for TART users and explains both input options to use and tools that are available to help users interpret results as relate to fission neutrons. Note that currently there is no delayed photon data generally available, and as such currently there is no treatment of delayed photons in TART. In TART the treatment of neutrons emitted in fission are controlled by,

\section{sentl 57}

Prompt neutrons are always emitted instantaneously in the prompt emission spectrum. This option controls how delayed neutrons are treated. Below I describe the treatment for each option,

$0=$ Delayed neutrons are emitted instantaneously in the prompt energy emission spectrum (this is the default).

1 = Delayed neutrons are ignored (this is appropriate for reactor accidents where there is a rapid flux excursion that is too fast for delayed neutrons to contribute). 
2 = Delayed neutrons are emitted in the delayed energy emission spectrum, but delayed emission is instantaneous (this is now the recommended option for criticality problems, where we assume the system has been running forever in a steady state, so that the fission products precursors are in equilibrium; delayed neutron emission time is not important, but emission energy may be important).

3 = Delayed neutrons are emitted in the delayed energy emission spectrum, and time dependent spectrum (this is now the recommended option for time dependent neutron source problems, where you are interested in the time dependent effect of delayed neutrons). This option is not allowed for criticality problems (use 2, as defined above).

\section{sentl 54}

If you are interested in neutron correlation studies, sentl 54 can be used to define how the number of neutrons per fission is defined. The default is to use the energy dependent average number of neutrons per fission, $\langle v(E)>$. You can use sentl 54 to instead sample the distribution $v(E)$ for the number of neutrons emitted; this option is not allowed for criticality problems.

\section{sentl 55}

If you are interested in neutron correlation studies, sentl 55 can be used to define a spontaneous fission sources; this is currently limited to $\mathrm{Pu}^{240}, \mathrm{Cm}^{242}, \mathrm{Cm}^{244}$, or $\mathrm{Cf}^{252}$; this option is not allowed for criticality problem.

\section{sentl 46}

This allows you to control that the detail to which energy dependent results are output by TART,

$0=80$ neutron energy tally bins (this is the default)

$1=165$ neutrons energy tally bins

$2=616$ neutron energy tally bins (I recommend this for most detail if you intend to use the utility codes pathc or paths; see below)

\section{timdist}

This option can be used to define a time dependent neutron source distribution; the default is that all source neutrons are emitted at time, $t=0$. The above example for material assay with a time dependent source used this option. In one case timdist 10.0 $1.0 \mathrm{~d}+10$, for a source uniform in time between 0 and 100 seconds ( $1.0 \mathrm{~d}+10$ shakes); in the other case timdist $10.9999 \mathrm{~d}+101.0 \mathrm{~d}+10$, for a source uniform in time between 99.99 and 100 seconds $(0.9999 \mathrm{~d}+10$ and $1.0 \mathrm{~d}+10$ shakes $)$.

centim 
VERY IMPORTANT - centim defines the maximum time to track particles; the default is 1 second, input in shakes as $1.0 \mathrm{~d}+8$. When using the delayed neutron time distribution (sentl 57 3) make sure this is a BIG number, e.g., 1.0d+20. If you do not do this all delayed neutrons emitted after 1 second will be ignored, and will not appear in any output tallies. This option can be used to define time bins for output, e.g., for output every second between 1 and 100 seconds $(1.0 \mathrm{~d}+8$ to $100.0 \mathrm{~d}+8$ in shakes) use the following input with time in shakes,

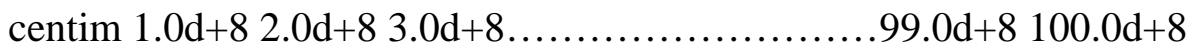

This is not the recommended way to obtain time dependent output; see the below description of ltype 12 and the TART utility code PLOT1112.

\section{Suggested Time Dependent Tallies: ltype 12}

If you wish you can define as input to TART many time bins in which to tally results (as described above), but this approach is time consuming, both for you to prepare the input and for TART to run, error prone, and not supported by any TART utility codes. A simpler approach is to use ltype $\mathbf{1 2}$ to tally results in binary files, that can later be processed by the utility code PLOT1112 to time and energy bin the results anyway you want; see the on-line TART document [6] for a description of ltype and the utility codes.

\section{Utility Codes}

All of the results presented in this report were obtaining using TART utility codes and this is what I suggest you use in your applications. Many of the utility codes are designed so that you never have to look inside the TART output file. Instead they can be used to convert TART results to PLOTTAB input format, so that you use the utility code PLOTTAB to can "see” your results,

BALANCE - was used to produce the energy dependent neutron production data.

PATHC - for criticality problems can be used to define neutron flux by spatial zone

PATHS - for source problems can be used to define neutron flux by spatial zone

PLOT1112 - was used to produce the time dependent neutron distribution.

PLOTTAB - can be used to plot output from any of these utility codes. 
University of California

Lawrence Livermore National Laboratory

Technical Information Department

Livermore, CA 94551

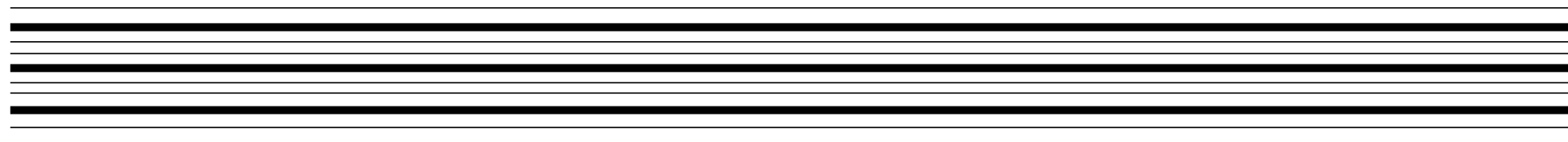

